\title{
Bernoulli shifts of the same entropy are finitarily and unilaterally isomorphic
}

\author{
ANDRÉS DEL JUNCO \\ Department of Mathematics, University of Toronto, Toronto, M5S 1A1, Canada
}

(Recelved 7 November 1988 and revised October 1989)

\begin{abstract}
Let $p=\left\{p_{1}, \quad, p_{n}\right\}$ and $q=\left\{q_{1}, \quad, q_{m}\right\}$ be finite probability vectors, each having at least three non-zero components, such that $-\sum_{t=1}^{n} p_{i} \log p_{t}=$ $-\sum_{i=1}^{m} q_{1} \log q_{1}$ Let $C=\{1, \quad, n\}, D=\{1, \quad, m\}$ and let $\left(C^{\mathbb{Z}}, p^{\mathbb{Z}}, \sigma\right)$ and $\left(D^{Z}, q^{Z}, \tau\right)$ be the corresponding Bernoullı shifts Then there exists an isomorphism $\phi$ between these shifts such that for a a $x \in C^{\mathbb{Z}} \phi(x)(0)$ is determined by finitely many of the future co-ordinates $x(0), x(1), \quad$ and for a a $y \in D^{\mathbb{Z}} \phi^{-1}(y)(0)$ is determined by finitely many of the co-ordinates $y(-1), y(0), y(1)$,
\end{abstract}

\section{Introduction}

Let $C=\{1, \quad, n\}$ and $D=\{1, \quad, m\}$ be finite sets Let $X=C^{\mathbb{Z}}$ denote the space of two-sided sequences indexed by $\mathbb{Z}$ and $Y=D^{\mathbb{Z}}$, both equipped with their Borel $\sigma$-algebras Suppose that $p$ and $q$ are probability measures on $C$ and $D$ Let $\mu=p^{\mathbb{Z}}$ and $\nu=q^{\mathbb{Z}}$ denote the corresponding product measures on $X$ and $Y$ The shift transformation $\sigma$ on $X$ is defined by $\sigma(x)(t)=x(l+1) \quad \tau$ will denote the shift on $Y(X, \mu, \sigma)$ and $(Y, \nu, \tau)$ are called Bernoulli shifts

A homomorphism from $(X, \mu, \sigma)$ to $(Y, \nu, \tau)$ is a measure-preserving map $\phi(X, \mu) \rightarrow(Y, \nu)$ such that $\phi \circ \sigma=\tau \circ \phi \mu$-a e $\phi$ is said to be finitary if $\forall j \in D$ $\phi^{-1}\{y \in Y \quad y(0)=\jmath\}$ agrees $\mu$-a e with a countable union of finite cylinder sets in $X$ Informally, for a a $x \in X \phi(x)(0)$ is determined by finitely many of the coordinates $\quad x(-1), x(0), x(1), \quad$ and, by shift invariance, the same is true of $\phi(x)(t)$ for all $t \in \mathbb{Z}$

We will call $\phi$ forgetful if for all $j \in D \phi^{-1}\{y \in Y \quad y(0)=\jmath\}$ agrees a $\mathrm{e}$ with a set in $\mathscr{F}^{+}$, the future $\sigma$-algebra in $X$, that is, the $\sigma$-algebra generated by the projections $x \mapsto x(l), t=0,1$, Informally, $\phi(x)(0)$ is determıned by $x(0), x(1), \quad$ We will call $\phi$ finitarily forgetful if for all $\jmath \in D \phi^{-1}\{y \in Y \quad y(0)=j\}$ agrees a $\mathrm{e}$ with a countable union of cylınder sets each of which is in $\mathscr{F}^{+}$It is an easy exercise that $\phi$ is finitarily forgetful if and only if it is finitary and forgetful

$\phi$ is called an isomorphism if it has an a e inverse $\psi$, that is $\psi \phi=\imath d$ a $\mathrm{e}$ The entropy $h(p)$ of $p$ is defined by

$$
h(p)=-\sum_{i=1}^{n} p_{i} \log p_{i}
$$


(We use $\log _{2}$ exclusively) The purpose of this paper is to prove the following result

THEOREM 1 If $h(p)=h(q)$ and $p$ and $q$ each have at least three non-zero components then there is a finitarly forgetful isomorphism $\phi$ from $(X, \mu, \sigma)$ to $(Y, \nu, \sigma)$ whose inverse is finitary

Except for the three-state assumption Theorem 1 is a strengthening of Keane and Smorodınsky's finitary ısomorphısm theorem for Bernoullı shıfts [K, S] The present paper is a continuation of the work in $[\mathbf{J}]$ where we established the existence of a finitarily forgetful homomorphism under the hypotheses of Theorem 1 Sinai [S] established the existence of a forgetful homomorphism under more general hypotheses ( $\mu$ any ergodic $\sigma$-invariant probability and $(Y, \nu, \tau)$ any Bernoullı shift with $h(\tau) \leq h(\sigma))$ Ornstein and Weiss [O, W] have given another proof of Sinai's theorem. Propp [P] has recently simultaneously generalızed Sinaı's theorem and given a proof entırely analogous to the proof of Ornstein's 1somorphism theorem [O], using the Baire category theorem as in $[\mathbf{B}, \mathbf{R}]$

If one removes all finitariness requirements on $\phi$ and $\phi^{-1}$, Theorem 1 asserts the existence of a forgetful isomorphism between $\sigma$ and $\tau$, which is still a new result We will call this weaker assertion Theorem 2 Because the proof of Theorem 1 is somewhat intricate we include here a considerably simpler proof of Theorem 2 usıng a Baire category argument The central idea of this proof is however the same as that of Theorem 1, namely the *-joining as defined in [J] In particular we observe here that under the right assumptions the *-operation has a certain symmetry which escaped notice in $[\mathbf{J}]$ and leads to a proof of the associativity of the *-operation

Theorem 1 falls short of its intended goal in two respects One of these is the troublesome three-state restriction The other is the natural conjecture that one should be able to make $\phi^{-1}$ causal, that is $\phi^{-1}(y)(0)$ depends only on the past $, y(-1), y(0)$ (It is well known [W] that $\phi$ and $\psi$ cannot both be forgetful unless $q$ is a re-arrangement of $p$ ) This conjecture is based on a natural desire for symmetry, but more cogently, on the fact that the desired symmetry is quite analogous to the aforementioned symmetry of the $*$-joining (In fact the $\phi^{-1}$ we obtain is in a certain sense close to being causal ) In this connection we mention Meshalkın's construction [M], in case $p=\left(\frac{1}{2}, \frac{1}{8}, \frac{1}{8}, \frac{1}{8}, \frac{1}{8}\right)$ and $q=\left(\frac{1}{4}, \frac{1}{4}, \frac{1}{4}, \frac{1}{4}\right)$, of a finitarily forgetful isomorphism with a finitarily causal inverse

As in $[\mathbf{K}, \mathbf{S}]$ and $[\mathbf{J}]$ the three-state assumption allows us to assume that $p_{1}=q_{1}$ As in $[K, S]$ and $[\mathbf{J}]$ we use the symbol 1 as a marker and $\phi$ reproduces in $y$ each occurrence of a marker in $x$ The $\phi^{-1}$ we obtain here is marker-conditionally causal in the sense that, once all occurrences of 1's in $y$ are known, then one need only look at $, y(-1), y(0)$ to determine $, x(-1), x(0)$

The plan of this paper is as follows $\S 2$ describes the function of markers $\S 3$ introduces the notion of a skeleton, its rank and its filler sets This section is very similar to $\S 3$ of $[\mathbf{J}]$ although somewhat streamlıned It contains most of the intricacies which are needed to achieve finitariness Roughly speakıng a skeleton $\mathscr{S}$ is a configuration of 1's (markers) and 0's (blanks) A skeleton can be filled by insertıng one of the symbols from $A=\{2, \quad, n\}(B=\{2, \quad, m\}$ in case of the $Y$ fillers $)$ in 
each blank spot For each $\mathscr{P}$ this is done only on a certain pre-assigned subset $I(\mathscr{P})$, in case of $X$-fillers, or $J(\mathscr{P})$, in case of $Y$-fillers, of the blank spots Later, in $\S 5$, we assign to each $\mathscr{S}$ a measure $\pi_{\mathscr{S}}$ on $A^{I(\mathscr{S})} \times B^{J(\mathscr{S})}$, whose marginals on $A^{I(\mathscr{S})}$ and $B^{J(\mathscr{S})}$ are $p_{0}^{I(\mathscr{C})}$ and $q_{0}^{J(\mathscr{S})}$, where $p_{0}$ and $q_{0}$ denote $p$ and $q$ conditioned on $A$ and $B$ respectively We call such a joint measure a superposition

In $\S 4$ we describe the *-joining which is essentially a way of combining two superpositions $\pi_{\mathscr{S}_{1}}$ and $\pi_{\mathscr{S}_{2}}$ to get a superposition $\pi_{\mathscr{S}_{1}} * \pi_{\mathscr{S}_{2}}$ which has both $\pi_{\mathscr{S}_{1}}$ and $\pi_{\varphi_{2}}$ as marginals It turns out that, in certain circumstances, the *-operation is associative Proposition 47 captures the key feature of the *-joining which makes it useful for coding roughly speaking, under the right assumptions, if $\pi_{\mathscr{S}_{n}}, \quad, \pi_{\mathscr{S}_{0}}, \pi_{\mathscr{S}_{-1}}$ are superpositions and $\pi_{\mathscr{S}_{-1}}$ is close to being a code from $A^{I\left(\mathscr{S}_{-1}\right)}$ to $B^{J\left(\mathscr{S}_{-1}\right)}$, in the sense that for most $x \in A^{I\left(\mathscr{S}_{-1}\right)} x$ is contained with respect to $\pi_{\mathscr{S}_{-1}}$ in some $y \in B^{J\left(\mathscr{S}_{-1}\right)} \quad$ (that is $\pi_{\mathscr{S}_{-1}}(x, y)=\pi_{\mathscr{S}_{-1}}\left(\{x\} \times B^{I\left(\mathscr{S}_{-1}\right)}\right)$ ), then $\pi_{\mathscr{S}_{n}} * \quad * \pi_{\mathscr{S}_{0}} * \pi_{\mathscr{S}_{-1}}$ is close to being a code no matter how far the $\pi_{\mathscr{S}_{1}}$ are from coding and how large $n$ is In [K, S] coding is achieved by taking the usual product measure $\pi_{\mathscr{S}_{n}} \times \quad \times \pi_{\mathscr{S}_{-1}}$ and then using a marriage lemma to perturb it in a way which respects any coding accomplished by the $\pi_{\mathscr{S}_{1}}$ and makes it close to coding However the perturbation no longer has the $\pi_{\mathscr{C}_{1}}$ as marginals The advantage of our approach is that no perturbation is required, which is what allows us to achieve forgetfulness

In $\S 5$ we define $\pi_{\mathscr{S}}$ for each $\mathscr{S}$ For $\mathscr{S}$ of odd rank we arrange matters so that by Proposition $47 \pi_{\mathscr{S}}$ is close to coding from $A^{I(\mathscr{S})}$ to $B^{J(\mathscr{S})}$ For even rank we make the coding go the other way The $\pi_{\mathscr{S}}$ are consistent in the sense that whenever $\overline{\mathscr{S}}$ is a subskeleton of $\mathscr{S} \pi_{\mathscr{S}}$ has marginal $\pi_{\mathscr{\mathscr { S }}}$ The consistency of the $\pi_{\mathscr{S}}$ allows us to combine them to obtain a joining of the Bernoull shifts $\sigma$ and $\tau$ and we are then able to show that this joining in fact arises from an isomorphism with the desired properties

In $\S 6$ we give the simpler proof of the non-finitary Theorem 2 It is essentially a much less careful version of the proof of Theorem 1 If the reader wants to read only Theorem 2 he should read $\S \S 2,4$ and 6 , which form a logically self-contained unit

\section{Markers}

Lemma 2 of $[\mathbf{K}, \mathbf{S}]$ enables us to assume that $p(1)=q(1)$ As in $[K, \mathbf{S}]$ the symbol 1 will be used as a marker in $X$ and $Y$, so we review some facts from [K, S] $X$ and $Y$ are fibred by the positions of marker occurrences as follows For $x \in X, \hat{x} \in \hat{X}=$ $\{0,1\}^{Z}$ is defined by

$$
\hat{x}(t)= \begin{cases}1 & \text { if } x(l)=1 \\ 0 & \text { otherwise }\end{cases}
$$

For $\xi \in \hat{X}, X(\xi)$ denotes the fibre over $\xi$

$$
X(\xi)=\{x \in X \quad \hat{x}=\xi\}
$$

The projection of $\mu$ onto $\hat{X}$, denoted by $\hat{\mu}$, is the product measure $\hat{p}^{\mathbb{E}}$ where $\hat{p}(1)=p(1), \hat{p}(0)=1-p(1)$ We make parallel definitions for $Y$ and evidently $\hat{\mu}=\hat{\nu}$ 
We denote by $\mu_{\xi}$ (respectively $\nu_{\xi}$ ) the conditional measure on $X(\xi)$ (respectively $\left.Y_{\xi}\right)$ so

$$
\mu=\int_{\hat{x}} \mu_{\xi} d \hat{\mu}(\xi)
$$

Settıng $A=\{2, \quad, n\}, B=\{2, \quad, m\}$ and $I(\xi)=\{t \in \mathbb{Z} \quad \xi(t)=0\}, X(\xi)$ is naturally identified with $A^{I(\xi)}$ and with this identification $\mu_{\xi}$ is $p_{0}^{I(\xi)}$ where $p_{0}$ denotes $p$ conditioned on $A$ Similarly $\nu_{\xi}$ is $q_{0}^{I(\xi)}$ where $q_{0}$ is $q$ conditioned on $B$

\section{Skeleta}

We denote an interval $\{l, l+1, \quad, \jmath\}$ in $\mathbb{Z}$ by $[l, \jmath],(l-1, \jmath],[l, j+1)$ or $(l-1, j+1)$ We will be dealıng with sequences $\gamma$ indexed by a subset $I$ of $\mathbb{Z}$ with entries chosen from a symbol set $\Gamma$ Thus formally $\gamma \in \Gamma^{\prime}$, that is $\gamma I \rightarrow \Gamma$ and $I$ is the domain of $\gamma$ For $I^{\prime} \subset I$ we will often denote the restriction of $\gamma$ to $I^{\prime}$ by $\gamma\left(I^{\prime}\right)$ (rather than $\gamma \mid I^{\prime}$ )

Let $N_{0}<N_{1}<N_{2}<$. be a sequence of positive integers to be specified later For $r \geq 0$ by a skeleton of rank $r$ we mean the pair $(r, \mathscr{S})$ where $\mathscr{S}$ is a sequence of 0 's (blanks) and 1's (markers) indexed by a finite interval $I$ in $\mathbb{Z}$ which has the form

$$
0^{m_{1}} 1^{n_{1}} 0^{m_{2}} 1^{n_{2}} \quad 0^{m_{k}} 1^{n_{h}}
$$

where $m_{1}>0, n_{1}>0$ and

$$
\max \{n, \quad 1 \leq l<k\}<N_{r} \leq n_{k}
$$

Thus any $r^{\prime}$ such that $\max \{n, 1 \leq \imath<k\}<N_{r^{\prime}} \leq n_{k}$ is a possible rank for the configuration (3 1) We distınguish between skeleta of different rank whose associated sequences are the same, even though we will usually speak loosely of the sequence $\mathscr{S}$ as a skeleton and write $r=\operatorname{rank} \mathscr{S}$ We will say $\mathscr{S}$ of the form $(31)$ has maximal rank if $r=\max \left\{r^{\prime} N_{r} \leq n_{k}\right\}$ We write

$$
|\mathscr{P}|=\{t \in I \quad \mathscr{S}(t)=0\},
$$

the set of blank indices of $\mathscr{S}$ We write $l(\mathscr{S})$ for $\#|\mathscr{P}|$

By a subskeleton $\overline{\mathscr{S}}$ of the skeleton $\mathscr{S}$ we mean the restriction of $\mathscr{S}$ to a subinterval $J$ or $I$ ending with a full marker run of $\mathscr{S}(1 \mathrm{e} \overline{\mathscr{S}}(1+\max J$ or $\max J=\max I)=0)$ such that $\overline{\mathscr{S}}$ is itself a skeleton with a rank not greater than that of $\mathscr{P}$ If $\overline{\mathscr{S}}$ is a subskeleton of $\mathscr{S}$ we write $\overline{\mathscr{S}}<\mathscr{S}$ For any $J \in|\mathscr{P}|$ the restriction of $\mathscr{S}$ to $I \cap[j, \infty)$ is a subskeleton of $\mathscr{S}$ with the same rank $r$ as $\mathscr{S}$ (Note that this is the only sort of restriction of $\mathscr{S}$ which may have a potential rank greater than $r$ ) Moreover every subskeleton of $\mathscr{S}$ of full rank is of this form We denote this subskeleton by ${ }_{f} \mathscr{S}$

A subskeleton $\overline{\mathscr{S}}$ of $\mathscr{S}$ will be called rank-maximal in $\mathscr{S}$ if it is maximal among the subskeleta of $\mathscr{S}$ with the same rank as $\overline{\mathscr{F}}$, ordered by $<$ Equivalently, the domain of $\overline{\mathscr{S}}$ cannot be extended to the left in $\mathscr{S}$ without increasing the rank of $\overline{\mathscr{S}}$, for the reason that $\overline{\mathscr{S}}$ is preceded immediately to the left by a marker run in $\mathscr{S}$ of length $l \geq N_{r}, r=\operatorname{rank} \mathscr{S}$

Subskeleta $\overline{\mathscr{S}}_{1}$ and $\overline{\mathscr{S}}_{2}$ of $\mathscr{S}$ may have overlappıng domains without one containıng the other, but the following lemma asserts that this cannot happen for rank-maximal subskeleta 
LEMMA 31 If $\overline{\mathscr{P}}_{1}$ and $\overline{\mathscr{P}}_{2}$ are rank-maximal subskeleta of $\mathscr{S}$ then either one is a sub-skeleton of the other or their domains are disjoint

Proof If dom $\overline{\mathscr{T}}_{1} \cap \operatorname{dom} \overline{\mathscr{S}}_{2} \neq \varnothing$ then (reversing the roles of $\overline{\mathscr{S}}_{1}$ and $\overline{\mathscr{T}}_{2}$ if necessary) the final marker block in $\overline{\mathscr{S}}_{1}$ is a marker block of $\overline{\mathscr{S}}_{2}$ It follows that the restriction of $\mathscr{S}$ to dom $\overline{\mathscr{S}}_{1} \cup \operatorname{dom} \overline{\mathscr{S}}_{2}$ is a subskeleton $\overline{\mathscr{S}}$ of $\mathscr{S}$ with the same rank as $\overline{\mathscr{S}}_{2}$ Since $\overline{\mathscr{S}}_{2}$ is rank maximal $\overline{\mathscr{S}}_{2}=\overline{\mathscr{S}}$ so dom $\overline{\mathscr{S}}_{1} \subset \operatorname{dom} \overline{\mathscr{S}}_{2}$ and min $\left(\operatorname{dom} \overline{\mathscr{S}}_{1}\right)=\min \left(\operatorname{dom} \overline{\mathscr{S}}_{2}\right)$ If $\max \left(\operatorname{dom} \overline{\mathscr{S}}_{1}\right)<\max \left(\operatorname{dom} \overline{\mathscr{S}}_{2}\right)$ we necessarly have rank $\overline{\mathscr{S}}_{1}<\operatorname{rank} \overline{\mathscr{S}}_{2}$ so $\overline{\mathscr{S}}_{1}<\overline{\mathscr{S}}_{2}$ In the other case $\operatorname{dom} \overline{\mathscr{S}}_{1}=\operatorname{dom} \overline{\mathscr{S}}_{2}$ so $\overline{\mathscr{S}}_{1}<\overline{\mathscr{S}}_{2}$ or $\overline{\mathscr{S}}_{2}<\overline{\mathscr{S}}_{1}$ according to which has the greater rank

If $\mathscr{S}$ is a skeleton of rank $r$ the occurrences of marker runs in $\mathscr{S}$ of length at least $N_{r-1}$ divide $\mathscr{S}$ into its rank-maxımal subskeleta of rank $r-1$ If these subskeleta are $\mathscr{S}_{1}, \quad, \mathscr{S}_{0}$ listed in order from left to right we write

$$
\mathscr{S}=\mathscr{S}_{1} \times \quad \times \mathscr{S}_{0}
$$

and refer to this as the rank decomposition of $\mathscr{S}$ (The apparent eccentricity in ordering, which will recur frequently, is in anticipation of the fact that all the constructions we shall make will have to be made from right to left in order to ensure forgetfulness ) Note that the rank decomposition may consist of $\mathscr{S}$ alone, with rank $r-1$ rather than $r$ We will make frequent use of induction on the rank of $\mathscr{S}$ and the fact that if $\overline{\mathscr{S}}<\mathscr{S}$ and rank $\overline{\mathscr{S}}<\operatorname{rank} \mathscr{S}$ then $\overline{\mathscr{S}}<\mathscr{S}$, for some $I$

Our next task is to define suitable subsets $I(\mathscr{S})$ and $J(\mathscr{S})$ of $|\mathscr{P}|$ so that $A^{I(\mathscr{S})}$ and $B^{J(\mathscr{S})}$ can play the role of filler sets First we define a method of truncating skeleta Let $0<C_{0}<C_{1} \quad$ be a sequence of positive integers to be specified later If $\mathscr{S}_{1}$ a 0 -skeleton define

$$
C(\mathscr{P})=\left.\right|_{\iota_{0}} \mathscr{S} \mid, \quad \iota_{0}=\min \left\{l \in|\mathscr{P}| l(, \mathscr{P}) \leq C_{0}\right\}
$$

Now suppose $C(\overline{\mathscr{S}})$ has been defined for all skeleta $\overline{\mathscr{S}}$ of rank less than $r$ and $\mathscr{S}$ has rank $r, \mathscr{S}=\mathscr{S}_{1} \times \quad \times \mathscr{S}_{0}$ Let

$$
\iota_{0}=\min \left\{l \in|\mathscr{S}| \quad l\left({ }_{1} \mathscr{S}\right) \leq C_{r}\right\}
$$

and suppose $t_{0} \in S_{\bar{i}}, t \geq \bar{t} \geq 0$ We define

$$
C(\mathscr{P})=\left.\right|_{\mathscr{H}_{0}} \mathscr{S} \mid \cup C\left(\mathscr{S}_{\bar{i}}\right)
$$

It is immediate by induction that the definition of $C(\mathscr{P})$ is forgetful in the sense that, for $\imath \in|\mathscr{S}|, C\left({ }_{1} \mathscr{S}\right)=C(\mathscr{S}) \cap[\imath, \infty)$ That 1 , in order to know how $C(\mathscr{S})$ looks to the right of $l$ one need only look at $\mathscr{S}$ to the right of $l$ It is also immediate that sor rank $\mathscr{S}=r$

$$
\# C(\mathscr{P}) \leq C_{0}+C_{1}+\quad+C_{r}
$$

If $I$ and $J$ are subsets of $\mathbb{Z}$ we write $I<J$ if $\max I<\min J$ The proof of the following lemma is immediate by induction on the rank of $\mathscr{S}$

LEMMA 32 If $\overline{\mathscr{S}}<\mathscr{S}$ then either $C(\overline{\mathscr{S}})<C(\mathscr{S})$ or $C(\overline{\mathscr{S}}) \subset C(\mathscr{P})$ 
If $\mathscr{G}$ is a skeleton we set

and

$$
R(\mathscr{S})=\{\max |\mathscr{P}|\}
$$

$$
C(\mathscr{P})=C(\mathscr{S})-R(\mathscr{P})
$$

We now proceed to define $I(\mathscr{S})$ and $J(\mathscr{S})$ If $\operatorname{rank}(\mathscr{S})=0$ set

$$
I(\mathscr{P})=J(\mathscr{P})=\varnothing
$$

Now let

and

$$
0<m_{1}<m_{2}<m_{3}<,
$$

$$
0<M_{2}<M_{4}<M_{6}<
$$

be sequences of positive integers to be specified later Suppose $\mathscr{S}=$ $\mathscr{S}_{t} \times \quad \times \mathscr{S}_{0} \times \mathscr{S}_{-1}$ and $r=\operatorname{rank} \mathscr{S}$ is odd Let us say $\mathscr{S}_{1}$ is initial in $\mathscr{S}$ if $0 \leq \imath<m_{r}$ and say $\mathscr{S}_{1}$ is principal in $\mathscr{S}_{\text {if }} \imath \geq m_{r}$ and $C\left(S_{t}\right) \subset C(\mathscr{S})$ Note that $\mathscr{S}_{-1}$ is neither initial nor principal Define

$$
\left.I(\mathscr{S})=\bigcup\left\{C\left(\mathscr{S}_{\imath}\right) \mathscr{S}_{1} \text { principal }\right\} \cup \bigcup R\left(\mathscr{S}_{1}\right) \mathscr{S}_{1} \text { inıtıal }\right\}
$$

and

$$
J(\mathscr{S})=\bigcup\left\{C\left(\mathscr{S}_{\imath}\right) \mathscr{S}_{1} \text { principal }\right\}
$$

Since $S_{-1}$ is not used we have $J(\mathscr{S}) \subset I(\mathscr{S}) \subset C(\mathscr{S})$

Now suppose that $\mathscr{P}=\mathscr{S}_{1} \times \quad \times \mathscr{S}_{0} \times \mathscr{S}_{-1}$ and $r=\operatorname{rank} \mathscr{S}$ is even For $0 \leq t \leq t$ let

$$
\imath=q\left(m_{r}+M_{r}\right)+s, \quad q \geq 0,0 \leq s<M_{r}+m_{r}
$$

and call $\mathscr{S}_{1}$ principal in $\mathscr{S}$ if $0 \leq s<M_{\text {r }}$ and $C\left(\mathscr{S}_{1}\right) \subset C(\mathscr{S})$ Call $\mathscr{S}_{1}$ auxiliary in $\mathscr{S}$ If $M_{r} \leq s<M_{r}+m_{r}$ and $C\left(\mathscr{S}_{1}\right) \subset C(\mathscr{S})$ In other words we skıp $\mathscr{S}_{-1}$ and then working from night to left we label the first $M_{r} \mathscr{S}_{i}$ 's principal, the next $m_{r}$ auxiliary and so on, as long as $C\left(\mathscr{S}_{1}\right)$ remains inside $C(\mathscr{P})$, after which we stop We will refer to any sequence

$$
\beta=\left(\mathscr{S}_{J+M_{r}+m_{r}-1}, \quad, \mathscr{S}_{j+1}, \mathscr{S}_{j}\right)
$$

of $m_{r}$ auxiliary $\mathscr{S}_{i}$ 's followed by $M_{r}$ principal $\mathscr{S}_{1}$ as a full block of $\mathscr{S}$ We set

$$
\begin{gathered}
|\beta|=\bigcup\left\{\left|\mathscr{S}_{\imath}\right| \jmath \leq \imath<\jmath+M_{r}+m_{r}\right\}, \\
J(\beta)=J(\mathscr{S}) \cap|\beta|=\bigcup\left\{R\left(\mathscr{S}_{\imath}\right) \quad J+M_{r} \leq \imath<\jmath+M_{r}+m_{r}\right\} \\
\cup \bigcup\left\{C\left(\mathscr{S}_{\imath}\right) \quad \leq \imath<M_{r}\right\}
\end{gathered}
$$

and

$$
\begin{aligned}
I(\beta) & =I(\mathscr{S}) \cap|\beta| \\
& =\bigcup\left\{C\left(\mathscr{S}_{1} J \leq 1<\jmath+M_{r}\right\}\right.
\end{aligned}
$$

Note that the forgetfulness of $C(\mathscr{S})$ implies the forgetfulness of $I(\mathscr{S})$ and $J(\mathscr{S})$ for $\imath \in|\mathscr{P}|$

$$
\begin{aligned}
& I\left({ }_{1} \mathscr{S}\right)=I(\mathscr{S}) \cap[l, \infty), \\
& J\left({ }_{1} \mathscr{S}\right)=J(\mathscr{S}) \cap[l, \infty)
\end{aligned}
$$

LEMMA 33

(a) If $\overline{\mathscr{S}}<\mathscr{S}$ then either $I(\overline{\mathscr{S}}) \cup J(\overline{\mathscr{S}}) \subset C(\mathscr{S})$ or

$$
I(\overline{\mathscr{S}}) \cup J(\overline{\mathscr{S}})<C(\mathscr{S})
$$


(b) If $\overline{\mathscr{S}}<\mathscr{S}$ then either

(1) $I(\overline{\mathscr{S}}) \subset I(\mathscr{S})$ and $J(\overline{\mathscr{S}}) \subset J(\mathscr{P})$ or

(11) $I(\overline{\mathscr{S}}) \cap I(\mathscr{P})=\varnothing$ and $J(\overline{\mathscr{P}}) \cap J(\mathscr{S})=\varnothing$

Proof

(a) Since $I(\overline{\mathscr{S}}) \cup J(\overline{\mathscr{S}}) \subset C(\overline{\mathscr{S}})$ (a) follows from Lemma 32

(b) If rank $\overline{\mathscr{S}}=\operatorname{rank} \mathscr{S}$ then $\overline{\mathscr{S}}={ }_{j} \mathscr{S}$ for some $j \in|\mathscr{S}|$ so $I(\overline{\mathscr{S}})=I(\mathscr{S}) \cap[\jmath, \infty) \subset I(\mathscr{S})$ and similarly $J(\overline{\mathscr{S}}) \subset J(\mathscr{S})$ Thus we may assume rank $\overline{\mathscr{S}}<$ rank $\mathscr{S}$ so $\overline{\mathscr{S}}<\mathscr{S}$, for some $\mathscr{S}_{1}$ in the rank decomposition $\mathscr{S}=\mathscr{S}_{1} \times \quad \times \mathscr{S}_{-1}$ We deal first with the case when rank $\mathscr{S}$ is odd If $\mathscr{S}_{t}$ is initial or $l=-1$ then $I(\overline{\mathscr{S}}) \cap I(\mathscr{S})=J(\overline{\mathscr{S}}) \cap$ $J(\mathscr{S})=\varnothing$ since $I(\overline{\mathscr{S}}) \cup J(\overline{\mathscr{S}}) \subset C\left(\mathscr{S}_{1}\right)$ If $\mathscr{S}_{1}$ is neither initial nor principal then certainly $I(\overline{\mathscr{S}}) \cap I(\mathscr{S})=J(\overline{\mathscr{S}}) \cap J(\mathscr{S})=\varnothing$ Finally if $\mathscr{S}_{1}$ is principal we have by (a) $I(\overline{\mathscr{S}}) \cup J(\overline{\mathscr{S}}) \subset C\left(\mathscr{S}_{1}\right)$ or $I(\overline{\mathscr{S}}) \cup J(\overline{\mathscr{S}})<C\left(\mathscr{S}_{1}\right)$ In the first case we actually have $I(\overline{\mathscr{S}}) \subset C\left(\mathscr{S}_{1}\right) \subset I(\mathscr{S})$ (since $\mathscr{S}_{1}$ is principal) and similarly $J(\overline{\mathscr{S}}) \subset J(\mathscr{S})$ In the second case evidently $I(\overline{\mathscr{S}}) \cap I(\mathscr{S})=J(\overline{\mathscr{S}}) \cap J(\mathscr{S})=\varnothing$

If rank $\mathscr{S}$ is even the argument is exactly the same, replacing 'initial' by 'auxiliary' throughout

Given a skeleton $\mathscr{S}$ we inductively define a famıly $D(\mathscr{S})$ of subskeleta of $\mathscr{S}$ as follows If rank $\mathscr{S}=0, D(\mathscr{S})=\varnothing$ If $\mathscr{S}=\mathscr{S}_{1} \times \mathscr{S}_{t-1} \times \quad \times \mathscr{S}_{0}$ we let

$$
D(\mathscr{S})=\{\mathscr{S}\} \cup \bigcup\left\{D\left(\mathscr{S}_{1}\right) \mathscr{S}_{1} \text { is not principal in } \mathscr{S}\right\} \text {. }
$$

LEMMA 34

(a) Each $\overline{\mathscr{S}} \in D(\mathscr{S})$ is rank-maximal in $\mathscr{S}$

(b) For distinct $\mathscr{S}_{1}, \mathscr{S}_{2} \in D(\mathscr{S}), I\left(\mathscr{S}_{1}\right) \cap I\left(\mathscr{S}_{2}\right)=J\left(\mathscr{S}_{1}\right) \cap J\left(\mathscr{Y}_{2}\right)=\varnothing$

(c) For each $\overline{\mathscr{S}}<\mathscr{S}$ there is a $\hat{\mathscr{S}} \in D(\mathscr{S})$ such that $\overline{\mathscr{S}}<\hat{\mathscr{F}}, J(\overline{\mathscr{S}}) \subset J(\hat{\mathscr{P}})$ and $J(\overline{\mathscr{S}}) \subset$ $J(\hat{\mathscr{S}})$

(d) The operator $D$ is forgetful for each $\imath \in|\mathscr{P}|$

$$
D(, \mathscr{S})=\left\{{ }_{1} \overline{\mathscr{S}} \overline{\mathscr{S}} \in D(\mathscr{S}), \imath \in|\overline{\mathscr{S}}|\right\} \cup\{\overline{\mathscr{S}} \in D(\mathscr{S}) \quad l<\min |\overline{\mathscr{S}}|\}
$$

Proof The proofs of (a), (b) and (d) are more or less immediate by induction To prove (c) suppose $\overline{\mathscr{S}}<\mathscr{S}$ If rank $\overline{\mathscr{S}}=\operatorname{rank} \mathscr{S}$ then $\overline{\mathscr{S}}={ }_{\imath} \mathscr{S}$ for some $\imath \in|\mathscr{S}|$ and we can take $\hat{\mathscr{S}}=\mathscr{S}$ If rank $\overline{\mathscr{S}}<$ rank $\mathscr{S}$ then $\overline{\mathscr{S}}<\mathscr{S}_{1}$ for some $\mathscr{S}_{1}$ in the decomposition $\mathscr{S}=\mathscr{S}_{t} \times \quad \times \mathscr{S}_{0}$ If $\mathscr{S}_{1}$ is principal then $I(\overline{\mathscr{S}}) \subset I(\mathscr{S})$ and $J(\overline{\mathscr{S}}) \subset J(\mathscr{S})$ so we can again take $\hat{\mathscr{S}}=\mathscr{S}$ If $\mathscr{S}_{1}$ is not principal then $D\left(\mathscr{S}_{1}\right) \subset D(\mathscr{S})$ so the result follows by induction

\section{The *-joining}

In this section we will be dealing with probability measures on various finite sets It will be convenient to adopt the following notational conventions for the rest of the paper If $E$ is a finite set and $P$ is the partition of $E$ into points, for any cartesian product $X$ in which $E$ is a factor $P$ will also denote the partition of that product according to the $E$-co-ordinate Thus for $p \in P, p$ denotes a subset of $E$ or of $X$ depending on the context

If $\rho$ is a probability measure on $X$ and $\alpha$ is a subset of $X, d_{\rho}(P \mid \alpha)$ will denote the conditional distribution of $P$ given $\alpha$, with respect to the measure $\rho$ Thus $d_{\rho}(P \mid \alpha)$ is in a natural way a measure $\rho$ on $E$ If $\alpha=X, d_{\rho}(P \mid \alpha)$ is the marginal of $\rho$ on $E$ and we denote it simply by $d_{\rho} P$ 
If $F$ is another finite set and $Q$ is the partition of $F$ into points then $P Q$ will denote the partition of $E \times F$ into points, which according to our convention is the supremum of the partitions $P$ and $Q$ on $E \times F$ If $p \in P$ and $q \in Q, p q \in P Q$ denotes the intersection of $p$ and $q$ considered as subsets of $E \times F$ Of course all these considerations extend to any product $X$ having $E \times F$ as a factor If $\rho$ and $\sigma$ are probability measures on $E$ and $F$ by a joinıng of $\rho$ and $\sigma$ we mean any measure $\lambda$ on $E \times F$ whose marginals are $\rho$ and $\sigma$ We write $P \perp Q(\lambda)$ if $\lambda=\rho \times \sigma$

Now suppose $\sigma$ and $\rho$ are probability measures on finite sets $F$ and $E$ each of which it totally ordered, so that

$$
F=\left\{f_{1}<f_{2}<\quad<f_{\mathrm{s}}\right\}, \quad E=\left\{e_{1}<e_{2}<<e_{r}\right\}
$$

We define a joinıng $\sigma \quad \rho$ of $\sigma$ and $\rho$ as follows Let

$$
0=y_{0}<y_{1}<\quad<y_{s}=1
$$

be points in $[0,1]$ such that

$$
\lambda\left(y_{1-1}, y_{1}\right)=\sigma\left(f_{t}\right) \text { for } 1 \leqslant \imath \leqslant s,
$$

where $\lambda$ denotes Lebesgue measure Similarly let

$$
0=x_{0}<x_{1}<\quad<x_{r}=1
$$

be such that

$$
\lambda\left(x_{\imath-1}, x_{1}\right)=\rho\left(e_{\imath}\right) \quad \text { for } 1 \leq \imath \leq r
$$

Define a jounıng $\sigma \quad \rho$ of $\sigma$ and $\rho$ by

$$
(\sigma \rho)\left(f_{j}, e_{t}\right)=\lambda\left(\left(y_{j-1}, y_{j}\right) \cap\left(x_{t-1}, x_{t}\right)\right)
$$

Denoting by $Q$ and $P$ the partitions of $F$ and $E$ into points, $\sigma \rho$ has the useful property that, in the joining $\sigma \quad \rho$, there are at most $\# F-1$ atoms $p \in P$ which are split by $Q$ (that is, $(\sigma \quad \rho)\left(p q_{1}\right)>0$ and $(\sigma \quad \rho)\left(p q_{2}\right)>0$ for distinct $\left.q_{1}, q_{2} \in Q\right)$

The proof of the following lemma is immediate from the definition of $\sigma \quad \rho$

LEMMA 41 Suppose $F, E_{2}$ and $E_{1}$ are finte totally ordered sets and $E_{2} \times E_{1}$ is given the lexicographic ordering $\left(e_{2}, e_{1}\right)<\left(e_{2}^{\prime}, e_{1}^{\prime}\right) \Leftrightarrow e_{2}<e_{2}^{\prime} \vee\left(e_{2}=e_{2}^{\prime} \wedge e_{1}<e_{1}^{\prime}\right)$ Let $Q, P_{2}$ and $P_{1}$ denote the partitions of $F, E_{2}$ and $E_{1}$ into points Suppose $\sigma$ and $\rho$ are probabilities on $F$ and $E_{2} \times E_{1}$ and let

$$
\gamma=\sigma \quad \rho, \quad \bar{\gamma}=d_{\gamma}\left(Q P_{2}\right),
$$

so $\bar{\gamma}$ is the marginal of $\gamma$ on $F \times E_{2}$ Then

$$
\bar{\gamma}=d_{\gamma}(Q) \quad d_{\gamma}\left(P_{2}\right)=d_{\sigma}(Q) \quad d_{\rho}\left(P_{2}\right),
$$

and for $p_{2} \in P_{2}$

$$
\begin{aligned}
& d_{\gamma}\left(Q P_{1} \mid p_{2}\right)=d_{\gamma}\left(Q \mid p_{2}\right) \quad d_{\gamma}\left(P_{1} \mid p_{2}\right) \\
& =d_{\tilde{\gamma}}\left(Q \mid p_{2}\right) d_{\rho}\left(P_{1} \mid p_{2}\right)
\end{aligned}
$$

Next suppose $\pi_{2}$ and $\pi_{1}$ are probability measures on $E_{2} \times F_{2}$ and $E_{1} \times F_{1}$ where $F_{2}$ and $E_{1}$ are totally ordered For $\iota=2,1 P_{1}$ and $Q_{1}$ denote the partitions of $E_{\imath}$ and $F$, into points We define a joinıng

$$
\pi=\pi_{2} * \pi_{1}
$$


of $\pi_{2}$ and $\pi_{1}$ on $\left(E_{2} \times E_{1}\right) \times\left(F_{2} \times F_{1}\right)$ by decreeing that

and

$$
\begin{gathered}
d_{\pi}\left(P_{2}\right)=d_{\pi_{2}}\left(P_{2}\right), \quad d_{\pi}\left(Q_{1}\right)=d_{\pi_{1}}\left(Q_{1}\right), \\
P_{2} \perp Q_{1} \quad(\pi)
\end{gathered}
$$

$$
d_{\pi}\left(Q_{2} P_{1} \mid p_{2} q_{1}\right)=d_{\pi_{2}}\left(Q_{2} \mid p_{2}\right) \quad d_{\pi_{1}}\left(P_{1} \mid q_{1}\right) \quad \forall p_{2} \in P_{2}, q_{1} \in Q_{1}
$$

Note that this is meaningful since we assumed $F_{2}$ and $E_{1}$ to be totally ordered

It will be important that the definition of $\pi_{2} * \pi_{1}$ is symmetric with respact to inter-changing the roles of $E_{1}$ and $F_{2}$ and of $E_{2}$ and $F_{1}$ To be more precise, if $\pi_{2}^{\prime}$ is the measure on $F_{2} \times E_{2}$ corresponding to $\pi_{2}$ under the natural identification of $F_{2} \times E_{2}$ with $E_{2} \times F_{2}$, and $\pi_{1}^{\prime}$ is the measure on $F_{1} \times E_{1}$ corresponding to $\pi_{1}$ then $\pi_{1}^{\prime} * \pi_{2}^{\prime}$ is meanıngful (since $F_{2}$ and $E_{1}$ are totally ordered) as a measure on $\left(F_{1} \times F_{2}\right) \times\left(E_{1} \times E_{2}\right)$ and corresponds to $\pi_{2} * \pi_{1}$ It is useful to keef the following picture of $\pi_{2} * \pi_{1}$ in mind

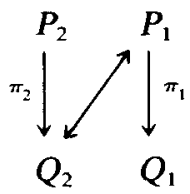

where the arrow indicates that, conditioned on any atom of $P_{2} Q_{1}, Q_{2}$ and $P_{1}$ are highly correlated, while $P_{2}$ and $Q_{1}$ are independent The symmetry we have just discussed should be viewed as symmetry under rotation of the picture by $180^{\circ}$

LEMMA $42 \pi=\pi_{2} * \pi$, is a joining of $\pi_{2}$ and $\pi_{1}$ Moreover with the above notation $P_{2} \perp P_{1} Q_{1}(\pi)$ and $Q_{1} \perp P_{2} Q_{2}(\pi)$

Proof Since

$$
d_{\pi}\left(Q_{2} P_{1} \mid p_{2} q_{1}\right)=d_{\pi_{2}}\left(Q_{2} \mid p_{2}\right) \quad d_{\pi_{1}}\left(P_{1} \mid q_{1}\right)
$$

and the dot-operation is a joining we have

$$
d_{\pi}\left(Q_{2} \mid p_{2} q_{1}\right)=d_{\pi_{2}}\left(Q_{2} \mid p_{2}\right)
$$

and averagıng over $q_{1}$

$$
d_{\pi}\left(Q_{2} \mid p_{2}\right)=d_{\pi_{2}}\left(Q_{2} \mid p_{2}\right)
$$

Since $d_{\pi}\left(P_{2}\right)=d_{\pi_{2}}\left(P_{2}\right)$ it follows that

$$
d_{\pi}\left(Q_{2} P_{2}\right)=d_{\pi_{2}}\left(Q_{2} P_{2}\right)
$$

that is $\pi$ has marginal $\pi_{2}$ By symmetry it also has marginal $\pi_{1}$ Moreover we have just observed that $Q_{2}$ and $Q_{1}$ are $P_{2}$-conditionally independent in the joining $\pi$ Since $Q_{1}$ and $P_{2}$ are independent it follows that $Q_{2} P_{2} \perp Q_{1}(\pi)$ By symmetry we also have $P_{2} \perp P_{1} Q_{1}(\pi)$

The next lemma asserts that under the right assumptions the *-operation is associative Suppose that $\pi_{1}$ is a probability on $E_{1} \times F_{1}$ for $l=3,2,1$ and that $F_{3}$, $F_{2}, E_{2}$ and $E_{1}$ are totally ordered Give $F_{3} \times F_{2}$ the reverse lexicographic ordering

$$
\left(f_{3}, f_{2}\right)<\left(f_{3}^{\prime}, f_{2}^{\prime}\right) \Leftrightarrow\left(f_{2}<f_{2}^{\prime}\right) \vee\left(f_{2}=f_{2}^{\prime} \wedge f_{3}<f_{3}^{\prime}\right),
$$


and put the usual lexicographic ordering on $E_{2} \times E_{1}$ Under these assumptions both $\pi_{3} *\left(\pi_{2} * \pi_{1}\right)$ and $\left(\pi_{3} * \pi_{2}\right) * \pi_{1}$ are defined on $\left(E_{3} \times E_{2} \times E_{1}\right) \times\left(F_{3} \times F_{2} \times F_{1}\right)$, since the appropriate sets are totally ordered

LEMMA 43 Under the above assumptions $\pi_{3} *\left(\pi_{2} * \pi_{1}\right)=\left(\pi_{3} * \pi_{2}\right) * \pi_{1}$

Proof As before, $P_{1}$ and $Q_{1}$ will denote the partitions of $E_{1}$ and $F_{1}$ into points We will show that $\pi=\pi_{3} *\left(\pi_{2} * \pi_{1}\right)$ has the following alternative description

$$
\begin{gathered}
d_{\pi}\left(P_{3} P_{2} Q_{2} Q_{1}\right)=d_{\pi}\left(P_{3}\right) \times d_{\pi}\left(P_{2} Q_{2}\right) \times d_{\pi}\left(Q_{1}\right) \\
d_{\pi}\left(Q_{3} P_{1} \mid p_{3} p_{2} q_{2} q_{1}\right)= \\
d_{\pi_{3} * \pi_{2}}\left(Q_{3} \mid p_{3} p_{2} q_{2}\right) \quad d_{\pi_{2} * \pi_{1}}\left(P_{1} \mid p_{2} q_{2} q_{1}\right), \\
\forall p_{3} \in P_{3}, p_{2} \in P_{2}, q_{2} \in Q_{2}, q_{1} \in Q_{1}
\end{gathered}
$$

Clearly (1) and (11) completely determıne $\pi$ The following picture of this description may be helpful

$$
\begin{gathered}
{\left[P_{3}\right]} \\
Q_{3}
\end{gathered}\left[\begin{array}{c}
P_{2} \\
Q_{2}
\end{array}\right] \begin{gathered}
P_{1} \\
{\left[Q_{1}\right]}
\end{gathered}
$$

The boxed partitions are jointly independent of each other Note that the above picture is symmetric under the rotation by $180^{\circ}$ Observing that rotation by $180^{\circ}$ interchanges the reverse and usual lexicographic orderings one sees that the same description is valid for $\left(\pi_{3} * \pi_{2}\right) * \pi_{1}$, establishing the lemma

To see that (1) holds observe that by Lemma $42 P_{3} \perp P_{2} P_{1} Q_{2} Q_{1}(\pi)$ and $P_{2} Q_{2} \perp Q_{1}$ $(\varepsilon)$, which implies (1)

As for (11), by Lemmas 41 and 42 and the definition of $\pi$ for $p_{3} \in P_{3}, q_{2} \in Q_{2}$, $q_{1} \in Q_{1}$ we have

$$
\begin{aligned}
& d_{\pi}\left(Q_{3} P_{2} \mid p_{3} q_{2} q_{1}\right)=d_{\pi}\left(Q_{3} \mid p_{3}\right) \quad d_{\pi}\left(P_{2} \mid q_{2} q_{1}\right) \\
& =d_{\pi_{3}}\left(Q_{3} \mid p_{3}\right) \quad d_{\pi_{2 *} \pi_{1}}\left(P_{2} \mid q_{2} q_{1}\right) \\
& =d_{\pi_{3}}\left(Q_{3} \mid p_{3}\right) \quad d_{\pi_{2}}\left(P_{2} \mid q_{2}\right)
\end{aligned}
$$

It follows that

$$
d_{\pi}\left(Q_{3} P_{2} \mid p_{3} q_{2}\right)=d_{\pi_{s}}\left(Q_{3} \mid p_{3}\right) \quad d_{\pi_{2}}\left(P_{2} \mid q_{2}\right)
$$

Since we also have $P_{3} \perp Q_{2}(\pi)$ we conclude that

$$
d_{\pi}\left(P_{3} P_{2} Q_{3} Q_{2}\right)=\pi_{3} * \pi_{2},
$$

that is $\pi$ has marginal $\pi_{3} * \pi_{2}$

Moreover the above calculation shows that $Q_{3} P_{2}$ and $Q_{1}$ are $P_{3} Q_{2}$-conditionally independent in the joining $\tau$ Since from (1) we already know we get $P_{3} Q_{2} \perp Q_{1}$ ( $\pi$ ) we get

$$
P_{3} P_{2} Q_{3} Q_{2} \perp Q_{1}(\pi)
$$

Finally by Lemma 41 and the definition of $\pi$ we have

$$
\begin{aligned}
& d_{\pi}\left(Q_{3} P_{1} \mid p_{3} p_{2} q_{2} q_{1}\right)=d_{\pi}\left(Q_{3} \mid p_{3} p_{2} q_{2} q_{1}\right) \quad d_{\pi}\left(P_{1} \mid p_{3} p_{2} q_{2} q_{1}\right) \\
& \left.=d_{\pi}\left(Q_{3} \mid p_{3} p_{2} q_{2}\right) \quad d_{\pi}\left(P_{1} \mid p_{2} q_{2} q_{1}\right) \text { (by (1v) and Lemma } 42\right) \\
& =d_{\pi_{3} * \pi_{2}}\left(Q_{3} \mid p_{3} p_{2} q_{2}\right) d_{\pi_{2 * \pi}}\left(P_{1} \mid p_{2} q_{2} q_{1}\right) \text { by (111), }
\end{aligned}
$$

whıch establıshes (11) 
If $I$ and $J$ are finite subsets of $\mathbb{Z}$ we denote by $\mu_{I}$ and $\nu_{J}$ the measures $p_{0}^{I}$ and $q_{0}^{J}$ on $A^{I}$ and $B^{J}$ respectively We denote by $P^{\prime}$ the partition of $A^{I}$ into points, so in keeping with our conventions $P^{\prime}$ is also a partition of $A^{\prime} \times B^{J}$ for any $J$ and $I^{\prime} \supset I Q^{\prime}$ will denote the partition of $B^{\prime}$ into points A probability measure $\pi$ on $A^{I} \times B^{J}$ will be called a superposition if it is a joining of $\mu_{I}$ and $\nu_{J}$ This includes the possibility that $I$ (or $J$ ) 1s empty then $\pi=\nu_{J}$ (or $\mu_{I}$ )

We now fix once and for all total orderings of $A$ and $B$ We endow $A^{I}$ with the lexicographic ordering and $B^{J}$ with the reverse lexicographic ordering for $y, y^{\prime} \in B^{J}$

$$
y<y^{\prime} \Leftrightarrow \exists_{J_{0}} \in J \text { s t } y\left(J_{0}\right)<y^{\prime}\left(J_{0}\right) \text { and } y(J)=y^{\prime}(J) \forall_{J}>J_{0}, J \in J
$$

Since any $A^{I}$ and $B^{J}$ are now totally ordered $\pi_{2} * \pi_{1}$ is defined whenever $\pi_{2}$ and $\pi_{1}$ are superpositions Moreover, since rotation by $180^{\circ}$ interchanges the usual and the reverse lexicographic orderıngs, $\pi_{2} * \pi_{1}$ is symmetric with respect to this rotation If $I_{3}<I_{2}<I_{1}$ and $J_{3}<J_{2}<J_{1}$ are subsets of $\mathbb{Z}$ and $\pi_{1}$ is a superposition on $A^{I_{i}} \times B^{J_{1}}$ then Lemma 43 implies that $\pi_{3} *\left(\pi_{2} * \pi_{1}\right)=\left(\pi_{3} * \pi_{2}\right) * \pi_{1}$ We will henceforth use this associativity without further comment and write simply $\pi_{3} * \pi_{2} * \pi_{1}$

A superposition $\pi$ on $A^{I} \times B^{J}$ will be called forgetful if for each $t \in \mathbb{Z}$

$$
P^{I \cap(-\infty 1)} \perp P^{I \cap[r \infty)} Q^{J \cap[t \infty)}(\pi)
$$

LEMMA 44 Suppose $I_{2}, I_{1}, J_{2}$ and $J_{1}$ are finite subsets of $\mathbb{Z}$ such that $I_{2} \cap I_{1}=\varnothing$ and $J_{2} \cap J_{1}=\varnothing$ and suppose that $\pi_{1}$ is a superposition on $A^{I_{1}} \times B^{J_{1}}(l=2,1)$ Then

(a) $\pi_{2} \times \pi_{1}$ and $\pi_{2} * \pi_{1}$ are superpositions on $A^{I_{2} \cup I_{1}} \times B^{J_{2} \cup J_{1}}$

(b) If $\pi_{2}$ and $\pi_{1}$ are forgetful then so is $\pi_{2} \times \pi_{1}$

(c) If there are subsets $K_{2}<K_{1}$ of $\mathbb{Z}$ such that $I_{1} \cup J_{1} \subset K_{1}(l=2,1)$ and $\pi_{2}$ and $\pi_{1}$ are forgetful then so is $\pi_{2} * \pi_{1}$

Proof

(a) $\pi_{2} \times \pi_{1}$ is obviously a superposition and $\pi_{2} * \pi_{1}$ is a superposition by Lemma 42

(b) $1 \mathrm{~s}$ easy

(c) Settıng $\pi=\pi_{2} * \pi$, we must show that

$$
P^{\left(I_{2} \cup I_{1}\right) \cap(-\infty t)} \perp P^{\left(I_{2} \cap I_{1}\right) \cap[t, \infty)} Q^{\left(J_{2} \cup J_{1}\right) \cap[t, \infty)} \quad(\pi),
$$

and we may as well assume that $t \in K_{2}$ or $t \in K_{1}$ If $t \in K_{1}$ (1) becomes

$$
P^{I_{2} \cup\left(I_{1} \cap(-\infty t)\right)} \perp P^{I_{1} \cap\left[f_{\infty} \infty\right)} Q^{J_{1} \cap[t \infty)} \quad(\pi),
$$

which is true because of Lemma 42 and because $\pi_{1}$ is forgetful If $t \in K_{2}$, (1) becomes

$$
P^{I_{2} \cap\left(-\infty I^{\prime}\right)} \perp P^{\left(I_{2} \cap[t, \infty)\right) \cup I_{1}} Q^{\left(J_{2} \cap[t, \infty)\right) \cup J_{1}}
$$

Let $p_{2}^{\prime} \in P^{I_{2} \cap(-\infty, t)}, p_{2}^{*} \in P^{\left(I_{2} \cap[1, \infty)\right.}$ and $q_{1} \in Q^{J_{1}}$ Because

$$
d_{\pi}\left(Q^{J_{2}} P^{I_{1}} \mid p_{2}^{\prime} p_{2}^{*} q_{1}\right)=d_{\pi_{2}}\left(Q^{J_{2}} \mid p_{2}^{\prime} p_{2}^{*}\right) \quad d_{\pi_{1}}\left(P^{I_{1}} \mid q_{1}\right)
$$

and because of Lemma 41 and the way $Q^{J_{2}}$ 1s ordered we see that

$$
d_{\pi}\left(Q^{J_{2} \cap[r, \infty)} P^{I_{1}} \mid p_{2}^{\prime} p_{2}^{*} q_{1}\right)=d_{\pi_{2}}\left(Q^{J_{2} \cap[r \infty)} \mid p_{2}^{\prime} p_{2}^{*}\right) d_{\pi_{1}}\left(P^{I_{1}} \mid q_{1}\right)
$$

Since $\pi_{2}$ is forgetful the distribution on the right above is independent of $p_{2}^{\prime}$, so the same is true of the distribution on the left Thus in the joining $\pi, Q^{J_{2} \cap(1 \infty)} P^{I_{1}}$ and $P^{I_{2} \cap(-\infty)}$ are $P^{I_{2} \cap\left[r^{\prime \infty}\right.} Q^{J_{1}}$-conditionally independent Since $P^{I_{2} \cap(-\infty \prime)} \perp P^{I_{2} \cap\left({ }^{\prime \infty)}\right.} Q^{\prime}$ ' by Lemma 42 , we conclude that (11) holds 
The following lemma may be viewed as an assertion of forgetfulness of the *-operation

LEMMA 45 Suppose that $\pi_{2}$ and $\pi_{1}$ are superpositions on $A^{I_{2}} \times B^{J_{2}}$ and $A^{l_{1}} \times B^{J_{1}}$ and that there are subsets $K_{2}<K_{1}$ of $\mathbb{Z}$ such that $I_{1} \cup J_{1} \subset K_{1}$ Fix $t \in K_{2}$ and let $\pi_{2}^{*}$ be the marginal of $\pi_{2}$ on $A^{I_{2} \cap[1, \infty)} \times B^{J_{2} \cap[1 \infty)}$ If $\pi_{2}$ is forgetful then the marginal of $\pi_{2} * \pi_{1}$ on $A^{\left(I_{2} \cap[1, \infty)\right) \cup I_{1}} \times B^{\left(J_{2} \cap[1, \infty)\right) \cup J_{1}}$ is $\pi_{2}^{*} * \pi_{1}$

Proof Adopting the notation in the proof of Lemma 44 (c) it follows from (111) in that proof and the forgetfulness of $\pi_{2}$ that

$$
d_{\pi}\left(Q^{J_{2} \cap\left[r^{\infty}\right)} P^{I_{1}} \mid p_{2}^{*} q_{1}\right)=d_{\pi_{2}^{*}}\left(Q^{J_{2} \cap[r, \infty)} \mid p_{2}^{*}\right) d_{\pi_{1}}\left(P^{I_{1}} \mid q_{1}\right)
$$

for all $p_{2}^{*} \in P^{I_{2} \cap[r \infty)}$ and $q_{1} \in Q^{J_{1}}$ We also have $P^{I_{2} \cap[r \infty)} \perp Q^{J_{1}}\left(\pi_{2} * \pi_{1}\right)$ since $P^{I_{2}} \perp Q^{J_{1}}$ $\left(\pi_{2} * \pi_{1}\right)$, so the result follows

Remark. The symmetry of $\pi_{2} * \pi_{1}$ allows one to conclude certain 'dual' statements from Lemmas 44 and 45 For example, the property dual to forgetfulness for a superposition $\pi$ is

$$
P^{I \cap(-\infty t]} Q^{J \cap(-\infty t]} \perp Q^{J \cap(t \infty)} \quad(\pi)
$$

Calling such a $\pi$ causal, Lemma $44(\mathrm{c})$ implies that, with the same assumptions on $I_{t}$ and $J_{t}$, if $\pi_{2}$ and $\pi_{1}$ are causal then so is $\pi_{2} * \pi_{1}$

We say a superposition $\pi$ on $A^{I} \times B^{J}$ splits $p \in P^{I}$ (or the corresponding $x \in A^{I}$ ) if $\pi(p q)>0$ and $\pi\left(p q^{\prime}\right)>0$ for distinct $q, q^{\prime} \in Q^{J}$ If this is not the case there is a $q \in Q^{\prime}$ such that $\pi(p q)=\pi(p)$ In this case we write $p \subset q(\pi)$ Analogous definitions apply to $q \in Q$

LEMMA 46 Suppose $I_{n}<I_{n-1}<\quad<I_{-1}$ are finte non-empty subsets of $\mathbb{Z}, J_{1} \subset I_{1}$ for $\imath=n, n-1, \quad, 0, \pi_{1}$ is a superposition on $A^{J} \times B^{J}$ for $t=n, n-1, \quad, 0$ and let $\pi_{-1}=\mu_{I_{-1}}$ on $A^{I_{-1}}$ For $\mathrm{J}=n, n-1, \quad,-1$ let

$$
\begin{aligned}
l_{j} & =\sum_{i=-1}^{J} \# I_{1}, \\
P^{\prime} & =P^{I_{j} \cup I_{,-1} \cup \cup I_{-1},} \\
\mu_{j} & =\mu_{I_{1} \cup I_{l-1} \cup \cup I_{-1},}
\end{aligned}
$$

and for $J=n, n-1, \quad, 0$ let

$$
\begin{aligned}
& Q^{\prime}=Q^{J} \cup \cup J_{0}, \\
& \nu_{J}=\nu_{J_{,} \cup \cup J_{0}}
\end{aligned}
$$

Let $h=h\left(p_{0}\right)=h\left(q_{0}\right)$ and fix $\varepsilon>0$ If $p \in P^{\prime}$ then $p=p^{\prime} p^{\prime-1} \quad p^{-1}$ with $p^{\prime} \in P^{\prime}$, Let us call $p$ good $f$

$$
\mu,(p)<2^{-(h-\varepsilon) !}
$$

Call $p$ completely good (cg) if $p^{\prime} p^{t-1} \quad p^{-1} \in P^{\prime}$ is good for all $\jmath \geq \imath \geq-1$ If $q^{\prime} \in Q^{\prime}$, for $\jmath \geq \imath \geq 0$, call $q=q^{\prime} q^{j^{-1}} \quad q^{0} \in Q^{\prime} \operatorname{good}$ if

$$
\nu_{J}(q)>2^{-(h-2 F) l}
$$

and completely good if $q^{1} \quad q^{0}$ is good for all $\jmath \geq \imath \geq 0$ For $n \geq J \geq-1$ let

$$
\Pi_{j}=\pi_{j} * \pi_{j-1} * \quad * \pi_{-1}
$$


Finally say that $p \in P^{\prime}$ is desirable if $p$ is not split by $\Pi_{,}, p \subset q\left(\pi_{J}\right)$ for a good $q \in Q^{\prime}$ and $p$ is completely good Then setting

$$
\rho_{j}=\mu_{j}\left(\bigcup\left\{p \in P^{\prime} p \text { is not desirable }\right\}\right),
$$

for $\jmath=n, n-1, \quad,-1$ we have

$$
\begin{aligned}
\rho_{J} \leq & \mu_{J}\left(\bigcup\left\{p \in P^{J} \quad p \text { is not c g }\right\}\right)+\nu_{J}\left(\bigcup\left\{q \in Q^{J} \quad q \text { is not cg }\right\}\right) \\
& +M \sum_{i=0}^{J} 2^{-\varepsilon(1+m)}
\end{aligned}
$$

where $M=\max _{0 \leq \imath \leq n} \# B^{J_{1}}-1, m=\# I_{0}$

Proof Notice that the definition of desirable is meanıngful for $p \in P^{-1}$ and that we trivially have the estımate

$$
\rho_{-1}<\mu \cup\left\{p \in P^{-1} p \text { is not } \mathrm{cg}\right\}
$$

To prove the lemma by induction it suffices to assume that the desired estimate on $\rho$, holds for $J=n-1$ and prove that it holds for $J=n$ For $p \in P^{n}$ write

$$
p=p^{n} p^{*} \quad \text { where } p^{n} \in P^{I_{n}}, p^{*} \in P^{n-1}
$$

We obviously have

where

$$
\rho_{n} \leq \rho_{n-1}+\mu_{n}(\bigcup \Gamma)
$$

$$
\Gamma=\left\{p^{n} p^{*} \in P^{n} p^{*} \text { is desirable but } p^{n} p^{*} \text { is not }\right\}
$$

We claim that

$$
\begin{aligned}
\mu_{n}(\bigcup \Gamma)< & M 2^{-\varepsilon(m+n)}+\mu_{n}\left(\bigcup\left\{\operatorname{bad} p^{n} p^{*} \in P^{n} p^{*} \text { is c g }\right\}\right) \\
& +\nu_{n}\left(\bigcup\left\{\operatorname{bad} q^{n} q^{*} \in Q^{n} q^{*} \text { is c g }\right\}\right),
\end{aligned}
$$

where bad means not good To see this first observe that if $p \in \Gamma$ then $p$ belongs to one of the following sets

$$
\begin{aligned}
& E_{1}=\Gamma \cup\left\{p \in P^{n} \quad p \text { is split by } \Pi_{n}\right\} \\
& E_{2}=\Gamma \cap\left\{p \in P^{n} \quad p \subset q\left(\pi_{n}\right), q \operatorname{good}\right\} \\
& E_{3}=\Gamma \cap\left\{p \in P^{n} \quad p \subset q\left(\pi_{n}\right), q \text { bad }\right\}
\end{aligned}
$$

We estımate the measure of $\bigcup E_{1}$ by regarding it as a union of atoms of $P^{n} \times Q^{n}$ and conditioning it on sets of the form $p_{1}^{n} q^{*}$ where $p_{1}^{n} \in P^{\prime n}, q^{*} \in Q^{n-1}$ In this argument all statements are to be interpreted modulo $\pi_{n}$-null sets Since $\bigcup E_{1}$ is contained in the union of completely good $Q^{n-1}$-atoms, when conditioning on $p_{1}^{n} q^{*}$ we may assume that $q^{*}$ is $\mathrm{cg}$ Fixing $p_{1}^{n} q^{*}$ if $p^{n} p^{*} \in E_{1}$ then, since $p^{*}$ is contained in a good $q \in Q^{n-1}$, either $p^{*} \subset q^{*}$ and $p^{n}=p_{1}^{n}$ or $p^{n} p^{*} \cap p_{1}^{n} q^{*}=\varnothing$ In the first case

$$
p^{*} \cap p_{1}^{n} q^{*}=p^{n} p^{*}=p_{1}^{n} p^{*}
$$

must be split by the partition $Q^{J_{n}} \cap p_{1}^{n} q^{*}$ of $p_{1}^{n} q^{*}$, for otherwise $p^{n} p^{*}$ would not be split by $Q^{n}$ Thus to estımate $\Pi_{n}\left(\bigcup E_{1} \mid p_{1}^{n} q^{*}\right)$ it suffices to estimate the 
$p_{1}^{n} q^{*}$-conditional measure of desirable $p^{*} \in P^{n-1}$ such that

Since

$$
p^{*} \subset q^{*} \text { and } p^{*} \cap p_{1}^{n} q^{*} \text { is split } Q^{\prime} \cap p_{1}^{n} q^{*}
$$

$$
d_{\Pi_{n}}\left(Q^{J_{n}} P^{n} \mid p_{1}^{n} q^{*}\right)=d_{\pi_{n}}\left(Q^{J} \mid p_{1}^{n}\right) \quad d_{\Pi_{n-1}}\left(P^{n} \mid q^{*}\right),
$$

there are at most \# $Q^{J_{1 \prime}}-1$ such $p^{*}$, and the conditional measure $\Pi_{n}\left(p^{*} \mid p_{1}^{n} q^{*}\right)$ of such a $p^{*}$ is

$$
\Pi_{n-1}\left(p^{*} \mid q^{*}\right) \leq 2^{-(h-\varepsilon) l_{n-1} / 2^{-(h-2 \varepsilon) l_{n-1}}=2^{-\varepsilon l_{n-1}},}
$$

since $p^{*} \subset q^{*}$ and both are completely good Thus

$$
\Pi_{n}\left(\bigcup E_{1} \mid p_{1}^{n} q^{*}\right) \leq\left(\# Q^{J_{n}}-1\right) 2^{-\varepsilon l_{n-1}} \leq M 2^{-\varepsilon(n+m)},
$$

whence also

$$
\Pi_{n}\left(\bigcup E_{1}\right)=\mu_{n}\left(\bigcup E_{1}\right) \leq M 2^{-\varepsilon(n+m)}
$$

Now if $p \in E_{2} p$ is bad, for otherwise $p$ would be desirable, so $\pi\left(\bigcup E_{2}\right)$ is less than the second term on the right of (11) $\pi\left(\cup E_{3}\right)$ is clearly less than the third term on the right of (11), which establishes (11) By (1), (11) and our induction hypothesis we have

$$
\begin{aligned}
\rho_{n} \leq & \mu_{n-1}\left(\bigcup\left\{p \in P^{n-1} \quad p \text { is not c g }\right\}\right)+\mu_{n}\left(\bigcup\left\{\operatorname{bad} p^{p} p^{*} \in P^{n} p^{*} \text { is c g }\right\}\right) \\
& +\nu_{n-1}\left(\bigcup\left\{q \in Q^{n-1} \quad q \text { is not c g }\right\}\right)+\nu_{n}\left(\bigcup\left\{\text { bad } q^{n} q^{*} \in Q^{n} q^{*} \text { is c g }\right\}\right) \\
& +M \sum_{t=0}^{n-1} 2^{-\varepsilon(t+m)}+M 2^{-\varepsilon(n+m)} \\
= & \mu_{n}\left(\bigcup\left\{p \in P^{n} p \text { is not c g }\right\}\right)+\nu_{n}\left(\bigcup\left\{q \in Q_{n} q \text { is not c g }\right\}\right) \\
& +M \sum_{t=0}^{n} 2^{-\varepsilon(t+m)}
\end{aligned}
$$

Lemma 46 is the property of the *-joining which is the key to the proof of Theorem 1 The following proposition articulates this property in a way which makes its applicability to coding clear

Proposition 47 Given $C \in \mathbb{Z}^{+}$and $\eta>0$ there exists $m \in \mathbb{Z}^{+}$such that the following holds Suppose $I_{n}<I_{n-1}<\quad<I_{0}<I_{-1}$ are finte subsets of $\mathbb{Z}$ such that

$$
\begin{aligned}
& \# I_{1} \leq C \quad \text { for } l \geq 0 \\
& \# I_{-1} \geq m,
\end{aligned}
$$

and $J_{1} \subset I_{1}$ for $1=n, \quad, 0$ are such that

$$
\# J_{1} \leq \# I_{1}-1
$$

Suppose $\pi_{1}$ is a superposition on $A^{I_{i}} \times B^{I_{i}}$ for $1=n, \quad, 0$ and $\pi_{-1}=\mu_{I_{-1}}$ Set

$$
\pi=\pi_{n} * \pi_{n-1} * \quad * \pi_{-1},
$$

and $I=I_{n} \cup I_{n-1} \cup \cup I_{0} \cup I_{-1}$ Then

$$
\mu_{1}\left\{x \in A^{\prime} x \text { is not split by } \pi\right\}>1-\eta
$$

Proof We adopt all the notation and terminology of Lemma 46 and also write

$$
\bar{l}_{j}=\sum_{i=0}^{J} \not J_{1}
$$


Since \# $I_{1} \leq C$ we have

$$
\# J_{1} \leq \# I_{1}-1 \leq\left(1-C^{-1}\right) \# I_{t}
$$

so

and

$$
\bar{l}_{j} \leq\left(1-C^{-1}\right) l
$$

$$
\max _{\mathfrak{l}} \# B^{J^{\prime}} \leq \# B^{C-1}=M
$$

Now fix $\varepsilon>0$ such that

$$
h-2 \varepsilon>\left(1-C^{-1}\right)(h+\varepsilon)
$$

By the a e Shannon-McMillan-Breiman theorem applied to the product measure $q_{0}^{N}$ (in fact in this setting all that is needed is the strong law of large numbers) we can find $k$ such that

$$
\forall K>k, \nu_{[1 K}\left\{y \in B^{[1, K]} \nu_{[1, \bar{I}]} y[1, \bar{l}]>2^{-(h+\varepsilon) \bar{l}} \text { for } k \leq \bar{l} \leq K\right\}>1-\eta
$$

(Recall that $y[1, \bar{l}]$ is the restriction of $y$ to $[1, \bar{l}]$ ) Next choose $m$ so that

$$
\begin{gathered}
\min \left\{\nu_{[1]}(y) \quad y \in B^{[1, \bar{l}]}, 0 \leq \bar{l} \leq k\right\}>2^{-(h-2 \varepsilon) m}, \\
\forall K \geq m, \mu_{[1 \kappa]}\left\{x \in A^{[1 K]} \mu_{[1 l]} x[1, l]<2^{-(h-\varepsilon) l} \text { for } m \leq l \leq K\right\}>1-\eta
\end{gathered}
$$

and

$$
M \sum_{i=m}^{\infty} 2^{-\varepsilon \imath}<\eta
$$

Now if $q=q^{n} \quad q^{0} \in Q^{n}$ is not completely good (for the $\varepsilon$ we have fixed) then for some $y \geq 0$

$$
\nu_{J}\left(q^{j} \quad q^{0}\right)<2^{-(h-2 \varepsilon) l_{j}}<2^{-(h-2 \varepsilon) m}
$$

(since $l_{j}=\# I_{j}+\quad+\# I_{-1} \geq \# I_{-1} \geq m$ ) so, by (111), $\bar{l}_{j}>k$ Moreover by (1)

$$
\nu_{j}\left(q^{j} \quad q^{0}\right)<2^{-(h-2 \varepsilon) l_{j}}<2^{-\left(1-C^{-1}\right)(h+\varepsilon) l_{j}}<2^{-(h+\varepsilon) \bar{l}_{j}}
$$

By (11) the $\nu_{n}$ measure of $q$ 's in $Q^{n}$ such that (v1) occurs for some $\bar{l}_{j}>k$ is less than $\eta$ so we have

(iv) implies that

$$
\nu_{n}\left(\bigcup\left\{q \in Q^{n} \quad q \text { is not } \mathrm{cg}\right\}\right)<\eta
$$

$$
\mu_{n}\left(\bigcup\left\{p \in P^{n} p \text { is not } \mathrm{cg}\right\}\right)<\eta
$$

In order to prove the lemma we may as well assume \# $I_{-1}=m$ Replacing $\eta$ by $\eta / 3$ the proposition now follows from (v), (v11), (v111) and Lemma 46

Proposition 48 Given $C \in \mathbb{Z}^{+}$and $\eta>0$ there is an $m \in \mathbb{Z}^{+}$such that the following hold Suppose $J_{-1}<J_{n}<J_{n-1}<\quad<J_{0}$ are finite subsets of $\mathbb{Z}$ such that

$$
\begin{aligned}
& \# J_{t} \leq C \quad l=n, n-1, \quad, 0 \\
& \# J_{-1} \geq m
\end{aligned}
$$

and $I_{1} \subset J_{l}, l=n, n-1, \quad, 0$ are such that

$$
\# I_{t} \leq \# J_{1}-1
$$


Suppose $\pi_{1}$ is a superposition on $A^{i} \times B^{J_{1}}$ for $1=n, n-1, \quad, 0$ and $\pi_{-1}=\nu_{J_{-1}}$ Set $\pi=\pi_{-1} * \pi_{n} * \pi_{n-1} * \quad * \pi$ and $J=J_{-1} \cup J_{n} \cup J_{n-1} \cup \cup J_{0}$ Then

$$
\nu_{J}\left\{y \in B^{J} \quad y \text { is not split by } \pi\right\}>1-\eta
$$

Proof This is a dual version of Proposition 47 which follows from the symmetry of the *-joining

\section{Construction of superpositions and proof of Theorem 1}

We now define for each skeleton $\mathscr{S}$ a superposition $\pi_{\mathscr{S}}$ on $A^{I(\mathscr{f})} \times B^{J(\mathscr{f})}$ Recall that If rank $\mathscr{S}=0$ then $I(\mathscr{S})=J(\mathscr{S})=\varnothing$ so there is nothıng to define Now suppose $\pi_{\varphi}$ has been defined when rank $\mathscr{S}<r$ and suppose $\mathscr{S}=\mathscr{S}_{1} \times \mathscr{S}_{t-1} \times \quad \times \mathscr{S}_{-1}$ has rank $r$ We deal first with the case of odd $r$ We assume $t \geq m_{r}$, as otherwise $J(\mathscr{S})=\varnothing$ so $\pi_{S}$ is simply $\mu_{I(\mathscr{S})}$ For each $t \geq 0$ such that $\mathscr{S}_{1+m_{r}}$ is principal let

and

$$
I_{t}=C\left(\mathscr{S}_{t+m_{r}}\right), \bar{I}_{i}=I_{t}-\bigcup\left\{I(\overline{\mathscr{S}}) \overline{\mathscr{S}} \in D\left(\mathscr{S}_{1+m_{r}}\right), I(\overline{\mathscr{S}}) \subset C\left(\mathscr{S}_{i+m_{r}}\right)\right\}
$$

$$
J_{i}=C\left(\mathscr{Y}_{1+m_{r}}\right), \bar{J}_{1}=J_{1}-U\left\{J(\overline{\mathscr{S}}) \overline{\mathscr{S}} \in D\left(\mathscr{S}_{1+m_{r}}\right), J(\overline{\mathscr{S}}) \subset C\left(\mathscr{S}_{1+m_{r}}\right)\right\}
$$

Define a superposition $\pi_{i}$ on $A^{\prime} \times B^{\prime}$, by

$$
\pi_{1}=\Pi\left\{\pi_{\overline{\mathscr{S}}} \overline{\mathscr{S}} \in D\left(\mathscr{S}_{1+m_{r}}\right)\right\} \times \mu_{\overline{I_{1}}} \times \nu_{\bar{J}_{1}}
$$

Note that this makes sense by Lemma $34(\mathrm{~b})$, (c) and is a superposition by Lemma 44 (a) In particular observe that when $\mathscr{S}$ has rank $1, \bar{I}_{i}=I_{i}, \bar{J}_{t}=J_{i}, D\left(\mathscr{S}_{1}\right)=\varnothing$ and $\pi_{1}=\mu_{I_{1}} \times \nu_{J_{1}}$ Now set

$$
I_{-1}=\bigcup\left\{R\left(\mathscr{P}_{1}\right) \quad 0 \leq l<m_{r}\right\}, \quad \pi_{-1}=\mu_{I_{-1}}
$$

and define $\pi_{\varphi}$ on $A^{I(f)} \times B^{J(\mathcal{Y})}$ by

$$
\pi_{\mathcal{S}}=\pi_{\bar{i}} * \pi_{\bar{i}-1} * \quad * \pi_{0} * \pi_{-1},
$$

where $\bar{t}$ 1s the largest $\imath$ such that $\mathscr{S}_{1+m_{r}}$ s principal $\pi_{f f}$ is a superposition by Lemma 44 (a) Note that $\pi_{f}$ has a structure of the type assumed in Lemma 47 Of course if $t<m_{r}$ then $J(\mathscr{S})=\varnothing$ so the conclusion of Lemma 47 holds vacuously

When $r=$ rank $\mathscr{S}$ is even we proceed in a sımilar manner as follows Suppose $\mathscr{S}=\mathscr{S}_{1} \times \quad \times \mathscr{S}_{-1}$ For each principal $\mathscr{Y}_{1}$ we set

$$
\begin{aligned}
& I_{t}=C\left(\mathscr{S}_{t}\right), \bar{I}_{t}=I_{t}-\bigcup\left\{I(\overline{\mathscr{S}}) \quad \overline{\mathscr{S}} \in D\left(\mathscr{S}_{1}\right), I(\overline{\mathscr{S}}) \subset C\left(\mathscr{S}_{1}\right)\right\} \text {, } \\
& J_{i}=C\left(\mathscr{P}_{i}\right), \bar{J}_{t}=J_{t}-\bigcup\left\{J(\overline{\mathscr{P}}) \quad \overline{\mathscr{P}} \in D\left(\mathscr{S}_{t}\right), J(\overline{\mathscr{S}}) \subset C\left(\mathscr{P}_{t}\right)\right\}
\end{aligned}
$$

and define a superposition $\pi_{1}$ on $A^{\prime} \times B^{J^{\prime}}$ by

$$
\pi_{1}=\Pi\left\{\pi_{\overline{\mathcal{F}}} \overline{\mathscr{S}} \in D\left(\mathscr{S}_{1+m_{r}}\right)\right\} \times \mu_{\bar{I}_{1}} \times \nu_{\bar{J}_{t}}
$$

For auxiliary $\mathscr{S}_{1}$ set $J_{i}=R\left(\mathscr{S}_{1}\right)$ and define $\pi_{1}=\nu_{R\left(f_{1}\right)}$ on $B^{J^{\prime}}$ Letting $\bar{t}$ denote the largest $l$ such that $C\left(\mathscr{S}_{1}\right) \subset C(\mathscr{S})$ define $\pi_{f}$ on $A^{(1)} \times B^{\prime(f)}$ by

$$
\pi_{f_{f}}=\pi_{\bar{i}} * \pi_{\bar{i}-1} \quad * \pi_{0}
$$

Note that if $\beta=\left(\mathscr{S}_{1}, \quad, \mathscr{S}_{\jmath}\right)\left(\imath=\jmath+M_{r}+m_{r}-1\right)$ is a full block of $\mathscr{P}$ then by associativity of the *-operation the marginal of $\pi$ on $A^{I(\beta)} \times B^{J(\beta)}$, which we will 
denote by $\pi_{\beta}$, is

where

$$
\begin{aligned}
\pi_{\beta}=d_{\pi}\left(P^{I(\beta)}, Q^{J(\beta)}\right) & =\left(\pi_{1} * \pi_{i-1} * \quad * \pi_{t-m_{r}+1}\right) * \pi_{t-m_{r}} * \quad * \pi_{J} \\
& =\pi_{-1} * \pi_{t-m_{r}} * * \pi_{J}
\end{aligned}
$$

$$
\pi_{-1}=\nu_{J_{-1}}, J_{-1}=R\left(\mathscr{S}_{1}\right) \cup R\left(\mathscr{S}_{t-1}\right) \cup \quad \cup R\left(\mathscr{S}_{\imath-m_{r}+1}\right)
$$

This $\pi_{\beta}$ has a structure of the type assumed in Proposition 48

For skeleta $\overline{\mathscr{S}}$ and $\mathscr{S}$ we will write $\overline{\mathscr{S}} \triangleleft \mathscr{S}$ if $\overline{\mathscr{S}}<\mathscr{S}, I(\overline{\mathscr{S}}) \cup J(\overline{\mathscr{S}}) \neq \varnothing, I(\overline{\mathscr{S}}) \subset I(\mathscr{S})$ and $J(\overline{\mathscr{S}}) \subset J(\mathscr{S})$ Lemma 33 (b) says that if $\overline{\mathscr{S}}<\mathscr{S}$ then either $\overline{\mathscr{S}} \triangleleft \mathscr{S}$ or $I(\overline{\mathscr{S}}) \cap$ $I(\mathscr{S})=J(\overline{\mathscr{S}}) \cap J(\mathscr{S})=\varnothing$ It is easy to see that $\overline{\mathscr{S}} \triangleleft \mathscr{S}$ if and only if $\overline{\mathscr{S}}=, \mathscr{S}$ for some $\jmath \in|\mathscr{P}|$ or $\overline{\mathscr{S}}<\mathscr{S}$, for some principal $\mathscr{S}$, in the rank decomposition of $\mathscr{S}$ and $I(\overline{\mathscr{S}}) \cup$ $J(\overline{\mathscr{S}}) \subset C\left(\mathscr{S}_{1}\right)$

LEMMA 51 The superpositions $\pi_{\mathscr{S}}$ are conststent in the sense that if $\overline{\mathscr{S}} \triangleleft \mathscr{S}$ then the marginal of $\pi_{\mathscr{S}}$ on $A^{I(\overline{\mathscr{S}})} \times B^{J(\overline{\mathscr{S}})}$ is $\pi_{\overline{\mathscr{S}}}$ The family $\left\{\pi_{\mathscr{S}}\right\}$ is translation invariant in the sense that if $\mathscr{S}^{\prime}$ is the shift of $\mathscr{S}$ then $\pi_{\mathscr{P ^ { \prime }}}$ is the shift of $\pi_{\mathscr{S}}$

Proof First observe that each $\pi_{\mathscr{S}}$ is forgetful, as can be seen by induction on rank $\mathscr{S}$ usıng Lemma 44 To prove the consistency assertıon by induction on rank $\mathscr{S}$ suppose $\overline{\mathscr{S}} \triangleleft \mathscr{S}$ If rank $\overline{\mathscr{S}}<$ rank $\mathscr{S}$ we must have $\overline{\mathscr{S}}<\mathscr{S}$, for some principal $\mathscr{S}_{\text {, }}$ in the rank decomposition of $\mathscr{S}$ and moreover $I(\overline{\mathscr{S}}) \cup J(\overline{\mathscr{S}}) \subset C\left(\mathscr{S}_{1}\right)$ By Lemma 34 (c) $\overline{\mathscr{S}} \triangleleft \mathscr{S}^{\prime}$ for some $\mathscr{S}^{\prime} \in D\left(\mathscr{S}_{1}\right)$ By induction $\pi_{\mathscr{S}}$ has marginal $\pi_{\mathscr{\varphi}}$ and by the definition of $\pi_{\mathscr{S}}$, $\pi_{\mathscr{S}}$ has marginal $\pi_{\mathscr{\varphi}}$, whence $\pi_{\varphi}$ has marginal $\pi_{\mathscr{S}}$ as required

Thus we may assume $\operatorname{rank} \overline{\mathscr{S}}=\operatorname{rank} \mathscr{S}$, so $\overline{\mathscr{S}}={ }_{\jmath} \mathscr{S}$ for some $J \in|\mathscr{S}|$ With $\mathrm{J}$ fixed, for any $I, J \subset \mathbb{Z}, \pi$ any measure on $A^{I} \times B^{J}$ and $\hat{\mathscr{S}}$ any skeleton such that $J \in|\hat{\mathscr{S}}|$ or $J<\min |\hat{\mathscr{P}}|$ let

$$
I^{*}=I \cap[J, \infty), \quad \pi^{*}=d_{\pi}\left(P^{I^{*}}, Q^{J^{*}}\right), \quad \hat{\mathscr{S}}^{*}= \begin{cases}J^{\hat{\mathscr{P}}} & \text { if } J \in|\hat{\mathscr{P}}| \\ \hat{\mathscr{S}} & \text { if } J<\min |\hat{\mathscr{P}}|\end{cases}
$$

What we must show is that $\left(\pi_{\mathscr{f}}\right)^{*}=\pi_{\varphi^{*}}$ Let $\mathscr{S}=\mathscr{S}_{1} \times \quad \times \mathscr{S}_{-1}$ and assume that rank $\mathscr{S}$ is odd for definiteness We may suppose $j \in\left|\mathscr{S}_{1+m_{r}}\right|, l \geq 0$ and $\mathscr{S}_{1+m_{r}}$ is principal, because if $j \in\left|\mathscr{S}_{k}\right|$ and $\mathscr{S}_{k}$ is not principal then what we are trying to show is trivial

Recall that

$$
\pi_{S}=\pi_{\bar{i}} * \pi_{\bar{i}-1} * \quad * \pi_{0} * \pi_{-1},
$$

where we adopt all the notation introduced in the definition of $\pi_{\mathscr{S}}$ Now

If we set

$$
S^{*}=\mathscr{P}_{i+m_{r}}^{*} \times S_{\imath-1+m_{r}} \times \quad \times S_{-1}
$$

$$
\bar{D}\left(\mathscr{S}_{r+m_{r}}\right)=\left\{\hat{\mathscr{S}} \in D\left(\mathscr{S}_{r+m_{r}}\right) \quad J \in|\hat{\mathscr{P}}| \text { or } j<\min |\hat{\mathscr{S}}|\right\}
$$

then Lemma 34 says that

$$
D\left(\mathscr{S}_{1+m_{r}}^{*}\right)=\left\{\hat{\mathscr{S}}^{*} \hat{\mathscr{S}} \in \bar{D}\left(\mathscr{P}_{1+m_{r}}\right)\right\}
$$

From this and the fact that $C\left(\mathscr{S}_{1+m_{r}}^{*}\right)=\left(C\left(\mathscr{S}_{t+m_{r}}\right)\right)^{*}$ one sees that

where

$$
\pi_{c, *}=\pi_{1}^{\prime} * \pi_{1-1} * \quad * \pi_{0} * \pi_{-1}
$$

$$
\pi_{1}^{\prime}=\Pi\left\{\left(\pi_{\hat{\dot{\varphi}} *} \hat{\mathscr{S}} \in \bar{D}\left(\mathscr{S}_{1+m_{r}}\right)\right\} \times \mu_{I_{i}^{*}} \times \nu_{\bar{J}_{*}^{*}}\right.
$$


Now by our induction hypothesis $\pi_{\hat{\mathscr{S}} *}=(\pi \hat{\mathscr{P}})^{*}$ for $\hat{\mathscr{S}} \in \bar{D}\left(\mathscr{Y}_{1+m_{r}}\right)$ so

and evidently

$$
\begin{aligned}
\Pi\left\{\pi_{\hat{\mathscr{S}} *} \hat{\mathscr{S}} \in \bar{D}\left(\mathscr{S}_{1+m_{r}}\right)\right\} & =\Pi\left\{\left(\pi_{\hat{\mathscr{S}}}\right)^{*} \hat{\mathscr{S}} \in \bar{D}\left(\mathscr{S}_{1+m_{r}}\right)\right\} \\
& =\left(\Pi\left\{\pi_{\hat{\mathscr{S}}} \hat{\mathscr{S}} \in D\left(\mathscr{S}_{1+m_{r}}\right)\right\}\right)^{*}
\end{aligned}
$$

$$
\mu_{I_{i}^{*}}=\left(\mu_{I_{1}}\right)^{*}, \quad \nu_{J_{1}^{*}}=\left(\nu_{J_{1}}^{*}\right)
$$

Thus $\pi_{\imath}^{\prime}=\pi_{\imath}^{*}$ Now $\pi_{\mathscr{S}}$ has marginal

$$
\pi_{1} * \pi_{1-1} * \quad \pi_{0} * \pi_{-1}
$$

and since $\pi_{1}$ is forgetful (Lemma $44(\mathrm{~b})$ and the fact that all $\pi_{\overline{\mathscr{S}}}$ are forgetful) by Lemma 45 this measure in turn has marginal

$$
\pi_{i}^{*} * \pi_{i-1} * \quad \cdot \pi_{-1}=\pi_{\mathscr{S} *}
$$

Thus $\pi_{\mathscr{S}}$ has marginal $\pi_{\mathscr{S}^{*}}$, which is what we wanted to prove

When $r$ is even the argument is essentially the same

Finally, the assertion about translation invariance is clear

Our next task is to construct for a a $\xi \in \hat{X}$ a superposition $\pi_{\xi}$ on $A^{I(\xi)} \times B^{I(\xi)}$ (that is $\pi_{\xi}$ is a joining of $\mu_{\xi}$ and $\nu_{\xi}$ ) whose marginal on $A^{I(\mathscr{P})} \times B^{J(\mathscr{C})}$ is $\pi_{\mathscr{S}}$ for every skeleton $\mathscr{S}$ occurring in $\xi$ (the precise meaning of this will be explained below)

For $\xi \in \hat{X}, \mathscr{S}$ a skeleton and $I=[l, J]$ an interval in $\mathbb{Z}$ we will say $\mathscr{S}$ occurs in $\xi$ on $I$ if $I=\operatorname{dom} \mathscr{S}$, the restriction $\xi(I)=\mathscr{S}$ and $\xi(J+1)=0$ We will say $\mathscr{S}$ is rank maximal in $\xi$ if no leftwards extension in $\xi$ of the domain of $\mathscr{S}$ yields a skeleton with the same rank as $\mathscr{S}$

Now suppose $\xi \in \hat{X}$ and each finite sequence of 0 's and 1's occurs infinitely often as a block in $\xi$ This is true for $\hat{\mu}$-a a $\xi$ Given such a $\xi$ and $r>0$ there exists a unique maximal interval $I$ in $\mathbb{Z}$ containg 0 such that $\xi(I)$ is an $r$-skeleton $I=\left[\iota_{0}, J_{0}\right]$, where

$$
\begin{aligned}
& l_{0}=\max \left\{l \in \mathbb{Z} \quad l \leq 0, \xi[l-N-r, l]=1^{N_{r}} 0\right\} \\
& J_{0}=\min \left\{j \in \mathbb{Z} \quad J \geq 0, \xi\left[J-N_{r}+1, j+1\right]=1^{N_{r}} 0\right\}
\end{aligned}
$$

We denote the $r$-skeleton $\xi(I)$ by $\mathscr{S}_{r}(\xi)$ Then $\mathscr{S}_{r}(\xi)$ occurs in $\xi$ on $I=\operatorname{dom} \mathscr{S}(\xi)$ and is rank-maximal in $\xi$ Evidently

$$
\mathscr{S}_{0}(\xi)<\mathscr{S}_{1}(\xi)<\mathscr{S}_{2}(\xi)<\quad,
$$

$\mathscr{S}_{r}(\xi)$ is rank-maximal in $\mathscr{S}_{r-1}(\xi)$ and

$$
\left|\mathscr{P}_{r}(\xi)\right| \nearrow I(\xi)
$$

We will also write

$$
I_{r}(\xi)=I\left(\mathscr{S}_{r}(\xi)\right) \quad J_{r}(\xi)=J\left(\mathscr{P}_{r}(\xi)\right)
$$

Having established this notation let us now fix a sequence $\eta_{r}>0$ such that $\sum \eta_{r}<\infty$ Fix an odd $r$ and suppose that $m_{t}, M_{t}, N_{1}$ and $C_{t}$ have been chosen for all $t<r$ (only for even $t$ in case of $M_{1}$ ) For any $r$-skeleton $\mathscr{S}=\mathscr{P}_{1} \times \quad \times \mathscr{Y}_{1}$ the sets $C\left(\mathscr{P}_{1}\right)$ 
are all bounded in size by $C_{0}+\quad+C_{r-1}$, so by Lemma 47 we can choose $m_{r}$ so that for all skeleta $\mathscr{S}$ of rank $r$

$$
\mu_{I(\mathscr{C})}\left\{x \in A^{I(\mathscr{S})} \pi_{\mathscr{S}} \text { splits } x\right\}<\eta_{r}
$$

Next we can choose $N_{r}$ and then $C_{r}$ so that

$$
\begin{gathered}
\hat{\mu}\left\{\xi \in \hat{X} \quad C\left(\mathscr{S}_{r-1}(\xi)\right) \subset I_{r}(\xi), C\left(\mathscr{S}_{r-1}(\xi)\right) \subset J_{r}(\xi),\right. \\
\left.\mathscr{S}_{r-1}(\xi) \text { is principal in } \mathscr{S}_{r}(\xi), \quad C\left(\mathscr{S}_{r}(\xi)\right)=\left|\mathscr{S}_{r}(\xi)\right|\right\}>1-\eta_{r}
\end{gathered}
$$

(Note that in fact the first two conditions in the definition of the above set are redundant ) To see that this is possible set

$$
G=\left\{\xi \in \hat{X} \quad \xi\left[0, N_{r-1}+2\right]=01^{N_{r-1}} 0\right\}
$$

and choose $K$ so large that the $\hat{\mu}$-measure of

$$
H_{1}=\left\{\xi \in \hat{X} \quad \sum_{i=0}^{k} 1_{G}\left((\hat{\sigma})^{i}(\xi)\right) \geq m_{r}+2\right\}
$$

1S greater than $1-\eta_{r} / 3$ ( $\hat{\sigma}$ denotes the shift on $\left.\hat{X}\right)$ Then settıng

$$
F=\left\{\xi \in \hat{X} \quad \xi\left[0, N_{r}-1\right]=1^{N_{r}}\right\}
$$

$N_{r}$ can be chosen so large that the $\hat{\mu}$-measure of

$$
H_{2}=\left\{\xi \in \hat{X} \quad \hat{\sigma}^{\prime}(\xi) \notin F \text { for } 0 \leq \imath \leq K\right\}
$$

is greater than $1-\eta_{r} / 3$ Finally, once $N_{r}$ has been chosen it is clear that $C_{r}$ can be chosen so large that the $\hat{\mu}$-measure of

$$
H_{3}=\left\{\xi \in \hat{X} \quad C\left(\mathscr{S}_{r}(\xi)\right)=\left|\mathscr{S}_{r}(\xi)\right|\right\}
$$

1s greater than $1-\eta_{r} / 3$ Now if $\xi \in H_{1} \cap H_{2}$ then $\mathscr{S}_{r-1}(\xi)$ is not initial in $\mathscr{P}_{r}(\xi)$ because there are at least $m_{r}+1$ rank maximal $r-1$ skeleta in $\mathscr{S}_{r}(\xi)$ to the right of $\mathscr{S}_{r-1}(\xi)$ If in addition $\xi \in H_{3}$ then all but the initial skeleta in the rank decomposition of $\mathscr{P}_{r}(\xi)$ are principal, and in particular $C\left(\mathscr{S}_{r-1}(\xi)\right) \subset I_{r}(\xi)$ and $C\left(\mathscr{P}_{r-1}(\xi)\right) \subset J_{r}(\xi)$ Since $\mu\left(H_{1} \cap H_{2} \cap H_{3}\right)>1-\eta_{r}$ we get 52

Now suppose that $r$ is even and $m_{i}, M_{\imath}, N_{\imath}$ and $C_{t}$ have been chosen for all $t<r$ By Lemma $48 m_{r}$ can be chosen so that for each $r$-skeleton $S$ and full block $\beta$ of $\mathscr{S}$

$$
\nu_{J(\beta)}\left\{y \in B^{J(\beta)} \pi_{\beta} \text { splits } y\right\}<\eta_{r}
$$

(Recall the remarks following the definition of $\pi_{\mathscr{S}}$ for even $\mathscr{S}$ )

Next we can choose $M_{r}, N_{r}$ and $C_{r}$, in that order, so that

$$
\begin{aligned}
\hat{\mu}\{\xi \in \hat{X} & \left.C\left(\mathscr{S}_{r-1}(\xi)\right)\right\} \subset J_{r}(\xi), C\left(\mathscr{S}_{r-1}(\xi)\right) \subset I_{r}(\xi), \\
& \mathscr{S}_{r-1}(\xi) \text { principal in } \mathscr{S}_{r}(\xi), \mathscr{S}_{r-1}(\xi) \text { belongs to } \\
& \text { a full block of } \left.\mathscr{S}_{r}(\xi), C\left(\mathscr{S}_{r}(\xi)\right)=\left|\mathscr{S}_{r}(\xi)\right|\right\}>1-\eta_{r}
\end{aligned}
$$

(Again the first two of the above conditions are redundant)

This is accomplished in much the same way as for odd $r$ First we choose $M_{r}$ much larger then $m_{r}$ and then we choose $N_{r}$ so that with high $\hat{\mu}$-probability the rank decomposition of $\mathscr{S}_{r}(\xi)$ contains $t r-1$-skeleta with $t>L\left(M_{r}+m_{r}\right)$ and $L$ is 
large In particular, with high probability $\mathscr{S}_{r-1}(\xi)$ will not be among the leftmost $M_{r} r-1$-skeleta in the rank decomposition of $\mathscr{S}_{r}(\xi)$ Finally $C_{r}$ is chosen so large that with high probability $\mathscr{S}_{r-1}(\xi)$ is principal in $\mathscr{S}_{r}(\xi)$ and also not among the leftmost $M_{r} r-1$-skeleta in $\mathscr{S}_{r}(\xi)$, which two conditions together ensure that $\mathscr{S}_{r-1}(\xi)$ belongs to a full block of $\mathscr{S}_{r}(\xi)$ The remaining conditions in (54) are immediate

We now assume that $m_{r}, M_{r}, N_{r}$ and $C_{r}$ have been chosen for all $r$ so that $(51)$ and (5 2) hold for all odd $r$ and (5 3) and (5 4) hold for all even $r$ By (5 2), (5 4) and the Borel-Cantelli lemma there is a set $\hat{X}^{\prime} \subset \hat{X}$ such that $\hat{\mu}\left(\hat{X}^{\prime}\right)=1$ and for each $\xi \in \hat{X}^{\prime}$ there is an $r_{0}(\xi)$ such that for $r \geq r_{0}(\xi) \xi$ belongs to the appropriate set in $(52)$ or (54) according as $r$ is odd or even Thus if $\xi \in \hat{X}^{\prime}$ then for sufficiently large odd $r$

$$
\left|\mathscr{S}_{r-1}(\xi)\right|-R\left(\mathscr{S}_{r-1}(\xi)\right)=C\left(\mathscr{P}_{r-1}(\xi)\right) \subset J_{r}(\xi) \subset I_{r}(\xi)
$$

Since $\left|\mathscr{S}_{r-1}(\xi)\right| \nearrow I(\xi)$ we conclude

$$
\bigcup_{r} J_{r}(\xi)=\bigcup_{r} I_{r}(\xi)=I(\xi)
$$

Moreover for sufficiently large $r$, even or odd, we have

$$
I_{r-1}(\xi) \subset C\left(\mathscr{S}_{r-1}(\xi)\right) \subset I_{r}(\xi),
$$

so the sequence $\left\{I_{r}(\xi)\right\}$ is eventually increasing, and similarly the same goes for $\left\{J_{r}(\xi)\right\}$ Thus for sufficiently large $r$ we have $\mathscr{S}_{r-1}(\xi) \triangleleft \mathscr{P}_{r}$. Whenever this is the case Lemma 51 implies that $\pi_{\mathscr{S}_{r-1}(\xi)}$ and $\pi_{\mathscr{S}_{r}(\xi)}$ are consistent measures and when it is not the case Lemma 33 (b) says that there is no conflict between $\pi_{\mathscr{C}_{r-1}(\xi)}$ and $\pi_{\mathscr{S}_{r}(\xi)}$

In view of these remarks for $\xi \in \hat{X}^{*}$ we can define $\pi_{\xi}$ to be the probability measure on $A^{I(\xi)} \times B^{I(\xi)}$ whose marginal on $A^{I_{r}(\xi)} \times B^{J_{r}(\xi)}$ is $\pi_{\mathscr{S}_{r}}$ for each $\xi$ It is clear that $\pi_{\xi}$ 1s a joinıng of $\mu_{\xi}$ and $\nu_{\xi}$ and that $\pi_{\xi}$ has marginal $\pi_{\mathscr{S}}$ for any skeleton $\mathscr{S}$ occurning in $\xi$, since $\mathscr{S}<\mathscr{S}_{r}(\xi)$ for sufficiently large $r$ Lemma 51 implies that the family $\left\{\pi_{\xi}\right\}$ is shift-invariant

$$
(\sigma \times \tau)\left(\pi_{\xi}\right)=\pi_{\hat{\sigma}(\xi)}
$$

(Recall that $A^{I(\xi)} \times B^{I(\xi)} \sim X(\xi) \times Y(\xi), \hat{\sigma}$ denotes the shift on $\hat{X}$ and $\sigma$ and $\tau$ the shifts on $X$ and $Y$, so $\sigma \quad X(\xi) \rightarrow X(\hat{\sigma}(\xi)))$ Now define $\pi$ on $X \times Y$ by

$$
\pi=\int_{\hat{X}} \pi_{\xi} d \hat{\mu}(\xi)
$$

(We leave it to the reader to formulate and verify the measurability which makes this meaningful ) $\pi$ is a joining of $\mu$ and $\nu$ and is invariant under $\sigma \times \tau$ The proof of Theorem 1 is now completed by the following proposition

Proposition 52 There exists a finitarly forgetful homomorphism $\phi \quad X \rightarrow Y$ with a finitary inverse $\psi$ such that for $B \subset X \times Y$

$$
\pi(B)=\mu\{x \in X \quad(x, \phi(x)) \in B\}
$$

Proof For $\xi \in \hat{X}^{\prime}$, define

$$
\phi_{\xi} X(\xi) \rightarrow Y(\xi)
$$


by requiring that, for $x \in X(\xi)$, the restriction $\phi_{\xi}(x)\left(J_{r}(\xi)\right)$ be $y_{r}$, whenever $x\left(I_{r}(\xi)\right) \subset$ $y_{r}\left(\pi_{\mathscr{S}_{r}(\xi)}\right)$

To check that this definition is unambiguous suppose that $r<r^{\prime}$,

and

$$
\begin{array}{ll}
x\left(I_{r}(\xi)\right) \subset y_{r} & \left(\pi_{\mathscr{S}_{r}(\xi)}\right), \\
x\left(I_{r^{\prime}}(\xi)\right) \subset y_{r} & \left(\pi_{\mathscr{S}_{r}}(\xi)\right),
\end{array}
$$

$$
S_{r}(\xi) \triangleleft S_{r}(\xi)
$$

(If the last condition does not hold then $I_{r}(\xi) \cap I_{r}(\xi)=J_{r}(\xi) \cap J_{r}(\xi)=\varnothing$, so there is no conflict between $y_{r}$ and $y_{r}$ ) Then the marginal of $\pi_{\mathscr{S}_{r}(\xi)}$ on $A^{I_{r}(\xi)} \times B^{J_{r}(\xi)}$ is $\pi_{\rho_{r}(\xi)}$ so, regarding the various finite sequences as cylınders in $A^{I_{r}(\xi)} \times B^{J_{r}(\xi)}$, up to $\pi_{\mathscr{P}_{r}(\xi)}$-null sets we have

and

$$
x\left(I_{r}(\xi)\right) \subset y_{r} \subset y_{r}\left(J_{r}(\xi)\right)
$$

Thus

$$
x\left(I_{r}(\xi)\right) \subset x\left(I_{r}(\xi)\right) \subset y_{r}
$$

$$
\begin{aligned}
\nu_{J_{r}(\xi)}\left(y_{r^{\prime}}\left(J_{r}(\xi)\right) \cap y_{r}\right) & =\pi_{\mathscr{f}_{r}(\xi)}\left(y_{r}\left(J_{r}(\xi)\right) \cap y_{r}\right) \\
& \geq \pi_{\mathscr{S}_{r}} x\left(I_{r}(\xi)\right)=\mu_{I_{r}(\xi)} x\left(I_{r^{\prime}}(\xi)\right)>0
\end{aligned}
$$

This means that, as cylinders in $B^{J_{r}(\xi)}, y_{r}\left(J_{r}(\xi)\right)=y_{r}$ (since otherwise they are disjoint), which is what we wanted to check

Next we must check that, given $\xi$, for $\mu_{\xi}$-a a $x \in X(\xi)$ the sequence $\phi_{\xi}(x)$ is defined on all of $I(\xi)$ By 51 , for odd $r$

$$
\mu_{I_{r}(\xi)}\left\{x \in A^{I_{r}(\xi)} \pi_{S_{r}(\xi)} \text { splits } x\right\}<\eta_{r}
$$

By Borel-Cantelli it follows that for $\mu_{\xi}$-a a $x \in X(\xi), x\left(I_{r}(\xi)\right)$ is split by $\pi_{S_{r}(\xi)}$ for only finitely many odd $r$ Thus for sufficiently large odd $r \phi_{\xi}(x)$ is defined on $J_{r}(\xi)$ and $\left\{J_{r}(\xi) \quad r\right.$ odd $\}$ is an eventually increasing sequence whose union is $I(\xi)$ This means that $\phi_{\xi}(x)$ is defined on $I(\xi)$ This completes the proof that the definition of $\phi_{\xi}$ is meaningful

The shift-invariance of $\left\{\pi_{\xi} \quad \xi \in \hat{X}\right\}$ evidently implies that the famıly $\left\{\phi_{\xi} \quad \xi \in \hat{X}\right\}$ is shift-ınvariant

$$
\phi_{\hat{\sigma}(\xi)} \circ \sigma=\tau \circ \phi_{\xi}
$$

We now define $\phi \quad X \rightarrow Y$ by

$$
\phi(x)=\phi_{\xi}(x) \quad \text { where } \xi=\hat{x}, 1 \text { e } x \in X(\xi)
$$

The shift invariance of $\left\{\phi_{\xi}\right\}$ implies that $\phi \circ \sigma=\tau \circ \phi$

In order to show that $\phi$ is finitarily forgetful we will define a one-sided version of $\phi$ We first introduce some one-sided notation Let

$$
X^{*}=C^{[0, \infty)}, \quad Y^{*}=D^{[0 \infty)}, \quad \hat{X}^{*}=\{0,1\}^{[0 \infty)}
$$

$x \mapsto \hat{x}$ denotes the natural projection $X^{*} \rightarrow \hat{X}^{*}$ and the same for $Y^{*} \rightarrow \hat{X}$ For $\xi \in \hat{X}^{*}$ let

$$
I^{*}(\xi)=\{l \quad \xi(l)=0\} \subset[0, \infty)
$$


Then

and

$$
X^{*}(\xi)=\left\{x \in X^{*} \hat{x}=\xi\right\} \sim A^{I^{*}(\xi)}
$$

$$
Y^{*}(\xi)=\left\{y \in Y^{*} \hat{y}=\xi\right\} \sim B^{I^{*}(\xi)}
$$

$x \mapsto x^{*}$ will denote the projection $X \rightarrow X^{*}$ and more generally also the projection from any two-sided sequence space to its one-sided version Thus for example if $\xi \in \hat{X}$ and $x \in X(\xi)$ then $\xi^{*} \in \hat{X}^{*}$ and $x^{*} \in X^{*}\left(\xi^{*}\right) \mu^{*}, \nu^{*}, \hat{\mu}^{*}, \mu_{\xi}^{*}$ and $\nu_{\xi}^{*}\left(\xi \in \hat{X}^{*}\right)$ denote the measures on $X^{*}, Y^{*}, \hat{X}^{*}, X^{*}(\xi)$ and $Y^{*}(\xi)$ naturally corresponding to $\mu, \nu, \hat{\mu}, \mu_{\xi}$ and $\nu_{\xi}$

For $\xi \in \hat{X}^{*}$ let $\imath_{0}(\xi)$ denote the least $\imath$ such that $\xi(\imath)=0$ As in the two-sided situation there exists for each $r$ a unique interval $I=\left[t_{0}(\xi), J\right]$ beginning with $t_{0}(\xi)$ such that $\xi(J+1)=0$ and $\xi(I)$ is an $r$-skeleton We denote this $r$-skeleton by $\mathscr{S}_{r}^{*}(\xi)$ and also write $I_{r}^{*}(\xi)=I\left(\mathscr{S}_{r}^{*}(\xi)\right)$ and $J_{r}^{*}(\xi)=J\left(\mathscr{S}_{r}^{*}(\xi)\right)$ For $\xi \in \hat{X}^{*}$ we define

$$
\phi_{\xi}^{*} X^{*}(\xi) \rightarrow Y^{*}(\xi)
$$

by requiring that, for $x \in X^{*}(\xi) \sim A^{I^{*}(\xi)}, \phi_{\xi}^{*}\left(J_{r}^{*}(\xi)\right)$ be $y_{r}$ whenever

$$
x\left(I_{r}^{*}(\xi)\right) \subset y_{r}\left(\pi_{\mathscr{S}_{r}^{*}(\xi)}\right)
$$

One argues that this defines the sequence $\phi_{\xi}^{*}(x) \in Y^{*}(\xi) \sim B^{I^{*}(\xi)}$ unambiguously and on all of $I^{*}(\xi)$, just as we did in the two-sided situation

Moreover for $\xi \in \hat{X}$ the mappings and $\phi_{\xi}$ and $\phi_{\xi^{*}}^{*}$ are consistent in the sense that for $x \in X(\xi)$

$$
\left(\phi_{\xi}(x)\right)^{*}=\phi_{\xi^{*}}\left(x^{*}\right)
$$

To see this just observe that for $\xi \in \hat{X}$ we must have

$$
\mathscr{S}_{r}^{*}\left(\xi^{*}\right)<\mathscr{S}_{r}(\xi)
$$

for all sufficiently large $r$ Since the skeleta have the same rank, $\mathscr{S}_{r}^{*}\left(\xi^{*}\right) \triangleleft \mathscr{S}_{r}(\xi)$ so the superpositions $\pi_{\mathscr{S}_{r}^{*}\left(\xi^{*}\right)}$ and $\pi_{\mathscr{S}_{r}(\xi)}$ are consistent This in turn allows us to argue just as in the two-sided situation that whenever

and

$$
x\left(I_{r}^{*}\left(\xi^{*}\right)\right) \subset y_{r}^{*} \quad\left(\pi_{\mathcal{S}_{r}^{*}\left(\xi^{*}\right)}\right)
$$

$$
x\left(I_{r}(\xi)\right) \subset y_{r} \quad\left(\pi_{\varphi_{r}(\xi)}\right)
$$

then $y_{r}^{*}$ is the restriction of $y_{r}$, which gives (1)

Now define $\phi^{*} X^{*} \rightarrow Y^{*}$ by

$$
\phi^{*}(x)=\phi_{\xi}^{*}(x) \quad \text { for } x \in X^{*}(\xi)
$$

$\phi^{*}$ is a finitary mapping since, for sufficiently large $r, \phi^{*}(x)\left(J_{r}(\xi)\right.$ ) (and hence $\left.\phi^{*}(x)(0)\right)$ is determined by $\pi_{\mathcal{C P}_{r}^{*}(\xi)}$ and $x\left(I_{r}^{*}(\xi)\right)$, both of which are determined by the restriction of $x$ to the domain of $\mathscr{S}_{r}(\xi)$ (1) implies that

$$
(\phi(x))^{*}=\phi^{*}\left(x^{*}\right),
$$

which in view of the finitariness of $\phi^{*}$ means that $\phi$ is finitarily forgetful

Next we show that for $B \subset X \times Y$

$$
\pi(B)=\mu\{x \quad(x, \phi(x)) \in B\}
$$


Fix $\xi \in \hat{X}$ and suppose that $p_{0} \in P^{I_{r^{0}}(\xi)}$ and $q_{0} \in Q^{J_{r^{0}}(\xi)}$ We recall the convention that $p_{0}$ and $q_{0}$ can also be regarded as subsets of larger Cartesian products By the definition of $\phi$ we have

$$
\begin{aligned}
\mu_{\xi}\{x & \left.\in X(\xi) \quad\left(x, \phi_{\xi}(x)\right) \in p_{0} q_{0}\right\} \\
& =\lim _{r \rightarrow \infty} \mu_{\xi}\left(\bigcup\left\{p \in P^{I_{r}(\xi)} p \subset p_{0}, \exists q \in Q^{J_{r}(\xi)} \text { st } p \subset q \subset q_{0}\left(\pi_{\mathscr{S}_{r}(\xi)}\right)\right\}\right) \\
& \leqslant \lim _{r \rightarrow \infty} \mu_{\xi}\left(\bigcup\left\{p \in P^{I_{r}(\xi)} p \subset p_{0}, p \subset q_{0}\left(\pi_{\mathscr{S}_{r}(\xi)}\right)\right\}\right) \\
& \leq \lim _{r \rightarrow \infty} \pi_{\mathscr{S}_{r}(\xi)}\left(p_{0} q_{0}\right)=\lim _{r \rightarrow \infty} \pi_{\mathscr{S}_{r}(\xi)}\left(p_{0} q_{0}\right)=\pi_{\xi}\left(p_{0} q_{0}\right)
\end{aligned}
$$

Thus whenever $B$ is a cylinder in $X(\xi) \times Y(\xi)$, and hence for all Borel $B \subset X(\xi) \times$ $Y(\xi)$, we have

$$
\mu_{\xi}\left\{x \in X(\xi)\left(x, \phi_{\xi}(x)\right) \in B\right\} \leq \pi_{\xi}(B)
$$

Now as a function of $B$ the left hand side of the above inequality is a probability measure on $X(\xi)$ Since $\pi_{\xi}$ is also a probability we can replace the inequality by equality Integratıng, we obtain (11) Note that one consequence of (11) is that $\phi$ is measure-preserving

It now remains only to construct a finitary $\psi \quad Y \rightarrow X$ inverse to $\phi$ For $\xi \in \hat{X}^{\prime}$ and even $r$ if $\mathscr{S}_{r-1}(\xi)$ lies in a full block in $\mathscr{S}_{r}(\xi)$ we denote this full block by $\beta_{r}(\xi)$ By (5 4) and the Borel-Cantelli lemma, for $\hat{\mu}$-a a $\xi, \beta_{r}(\xi)$ is defined once $r$ is sufficiently large and even Whenever $r<r^{\prime}$ and $\beta_{r}(\xi)$ and $\beta_{r}(\xi)$ are defined we have $\left|\beta_{r}(\xi)\right| \subset$ $\left|\beta_{r}(\xi)\right|$ and either

$$
\mathscr{S}_{r}(\xi) \triangleleft \mathscr{S}_{r}(\xi)
$$

or

$$
I_{r}(\xi) \cap I_{r}(\xi)=J_{r}(\xi) \cap J_{r}(\xi)=\varnothing
$$

In case (111) holds

$$
I\left(\beta_{r}(\xi)\right)=I_{r}(\xi) \cap\left|\beta_{r}(\xi)\right| \subset I_{r}(\xi) \cap\left|\beta_{r}(\xi)\right|=I\left(\beta_{r}(\xi)\right)
$$

and similarly $J\left(\beta_{r}(\xi)\right) \subset J\left(\beta_{r}(\xi)\right)$ Thus in case (111), $\pi_{\beta_{r}(\xi)}$, a measure on $A^{I\left(\beta_{r}(\xi)\right)} \times$ $B^{J\left(\beta_{r}(\xi)\right)}$, has marginal $\pi_{\beta_{r}(\xi)}$, since each is a marginal of $\pi_{\xi}$ In case (iv) we have

$$
I\left(\beta_{r}(\xi)\right) \cap I\left(\beta_{r}(\xi)\right)=J\left(\beta_{r}(\xi)\right) \cap J\left(\beta_{r^{\prime}}(\xi)\right)=\varnothing
$$

We have observed earlier that for a a $\xi$ (111) holds once $r$ is sufficiently large Moreover if $S_{r-1}(\xi)$ is principal in $S_{r}(\xi)$, which is the case for sufficiently large $r$ by $(54)$, we will have

$$
I\left(\beta_{r}(\xi)\right) \supset C\left(S_{r-1}(\xi)\right)
$$

Also if $r$ is sufficiently large 54 implies

$$
\left|S_{r-1}(\xi)\right|-R\left(S_{r-1}(\xi)\right)=C\left(S_{r-1}(\xi)\right),
$$

and since $\bigcup_{r}\left|S_{r-1}(\xi)\right|=I(\xi)$ we conclude $\bigcup_{r} I\left(\beta_{r}(\xi)\right)=I(\xi)$

We now define

$$
\psi_{\xi} \quad Y(\xi) \rightarrow X(\xi)
$$


by requiring that $\psi_{\xi}(y)\left(I\left(\beta_{r}(\xi)\right)\right)=x_{r}$ whenever $y\left(J\left(\beta_{r}(\xi)\right)\right) \subset x_{r} \quad\left(\pi_{\beta_{r}(\xi)}\right)$

together with the remarks in the previous paragraph allow us to conclude, as we did for $\phi_{\xi}$, that for $\nu_{\xi}$-a a $y \psi_{\xi}(y)$ is unambiguously defined on all of $I(\xi)$ We define $\psi \quad Y \rightarrow X$ by

$$
\psi(y)=\psi_{\xi}(y) \quad \text { where } y \in Y(\xi) \quad \text { i e } \hat{y}=\xi
$$

$\psi$ is finitary since for sufficiently large even $r \psi(y)$ is determined on $I\left(\beta_{r}(\hat{y})\right)$, and hence at 0 , by $y\left(J\left(\beta_{r}(\hat{y})\right)\right)$ and $\pi_{\beta,(\hat{y})}$ both of which are determined by the restriction of $y$ to the domain of $S_{r}(\hat{y})$, a finite portion of $y$ (However, see the remark after the end of this proof )

Finally for $B \subset X \times Y$ we obtain

$$
\pi(B)=\nu\{y \in Y \quad(\psi(y), y) \in B\}
$$

in the same way that we obtained (11) (v) implies that $\psi$ is measure-preserving (11) and (v) together imply that $\psi=\phi^{-1}$ for $E \supset X$

$$
\begin{aligned}
\mu\left\{\phi^{-1} \psi^{-1} E \cap E\right\} & =\mu\left\{x \in X \quad(x, \phi(x)) \in E \times \psi^{-1} E\right\} \\
& =\pi\left(E \times \psi^{-1} E\right) \\
& =\nu\left\{y(\psi y, y) \in E \times \psi^{-1} E\right\} \\
& =\nu\left(\psi^{-1}(E)\right)=\mu(E)
\end{aligned}
$$

Since $\psi \circ \phi$ is measure-preserving this means $\mu\left(E \Delta \phi^{-1} \psi^{-1} E\right)=0$, so $\psi \phi=\imath d$ a e

Remark. Referring to the remark after the proof of Lemma 45 it is not hard to see that the $\pi_{S}$ are all causal Recall that

$$
\pi_{\beta_{r}(\xi)}=\nu_{I_{-1}} * \pi_{i} * \pi_{i-1} * \quad * \pi_{j}
$$

where $\#\left(I_{-1}\right)=m_{r}$ and $l-\jmath=M_{r}$, and $\pi_{l}, \quad, \pi_{j}$ are products of lower rank $\pi_{\overrightarrow{\mathcal{S}}}$, and hence all causal The dual version of Lemma 45 then implies that the marginal of $\pi_{\beta_{r}(\xi)}$ on $A^{I\left(\beta_{r}(\xi)\right) \cap(-\infty, 0]} \times B^{\left.J\left(\beta_{r}(\xi)\right) \cap-(\infty) 0\right]}$, which we denote ${ }^{*} \pi_{\beta_{r}(\xi)}$, has the form

$$
* \pi_{\beta_{r}(\xi)}=\nu_{I_{-1}} * \pi_{i} * \pi_{t-1} * \quad * * \pi_{t},
$$

where ${ }^{*} \pi_{t}$ denotes the marginal of $\pi_{t}$ on the past Thus Proposition 48 applies to ${ }^{*} \pi_{\beta_{r}(\xi)}$ as well as $\pi_{\beta_{r}(\xi)}$ This leads to the observation that we can determine $\psi_{\xi}(y)(-\infty, 0]$ if we know $y(-\infty, 0)$ and ${ }^{*} \pi_{\beta_{r}(\xi)}$ for all $r$ Since ${ }^{*} \pi_{\beta_{r}(\xi)}$ is certainly determined by $\xi$ it follows that $\psi$ is marker-conditionally causal in the sense that once $\xi=\hat{y}$ is known then the past of $\psi(y)$ depends only on the past of $y$ However, one needs to know all of $\xi$ because ${ }^{*} \pi_{\beta_{r}(\xi)}$ is not determined by the past of $\xi$ alone Indeed, since auxiliary and princıpal skeleta are determıned by workıng from right to left, if we lose the right end of $\mathscr{S}_{r}(\xi)$ we will not even know what the domain of $* \pi_{\beta_{r}(\xi)}$ is

\section{Proof of Theorem 2}

The purpose of this section is to sketch a proof of Theorem 2, the non-finitary version of Theorem 1 Technical detalls will for the most part be suppressed We assume familiarity with $\$ \$ 2$ and 4 
THEOREM 2 There exists a forgetful isomorphism $\phi \quad X \rightarrow Y$ such that $\phi \quad X(\xi) \rightarrow Y(\xi)$

We continue to assume that $p(1)=q(1)$ and we maintain all the notation introduced in $\S \S 2$ and 4 Also extend that notation as follows If $I$ is finite we identify the partition $P^{I}$ (or $Q^{I}$ ) on any space having $A^{I}$ as a factor with the finite $\sigma$-algebra it generates If $I$ is infinite $P^{I}$ denotes the $\sigma$-algebra generated by the projection $X \rightarrow A^{\prime}, A^{\prime}$ being given the usual product Borel structure We recall also one notational eccentricity from $\S 3$ when $x$ is a sequence indexed by $\mathbb{Z}$ and $I \subset \mathbb{Z}, x(I)$ denotes the restriction of $x$ to $I$

If $(Z, \mathscr{B}, \lambda)$ is a probability space, $\mathscr{F}$ is a sub- $\sigma$-algebra of $\mathscr{B}$ and $R$ is a finite $\mathscr{B}$-measurable partition of $Z$ we write $R \subset \mathscr{F}(\lambda)$ if each $r \in R$ agrees a e with an $r^{\prime} \in \mathscr{F}$ For $\eta>0$ we write $R \stackrel{E}{\subset} \mathscr{F}(\lambda)$ if for each $r \in R$ there is an $r^{\prime} \in \mathscr{F}$ such that $\lambda\left(r \Delta r^{\prime}\right)<\eta$ It is not hard to see that if $Q$ and $R$ are finite partitions of $Z$ and

$$
\lambda(\bigcup\{q \in Q \quad \exists r \in R \text { s t } q \subset r \lambda \text {-a e }\})>1-\eta
$$

then $R \stackrel{\eta}{\subset} \sigma(Q)(\lambda)(\sigma(Q))$ denotes the $\sigma$-algebra generated by $Q)$

To prove Theorem 2 we must construct a measurably varyıng famıly $\left\{\phi_{\xi} \quad \xi \in \hat{X}\right\}$ of measurable and measure-preserving mappings $\phi_{\xi} X(\xi) \rightarrow Y(\xi) \phi_{\xi}$ corresponds to a joining $\pi_{\xi}$ of $\mu_{\xi}$ and $\nu_{\xi}$ for $B \subset X(\xi) \times Y(\xi)$

$$
\pi_{\xi}(B)=\mu_{\xi}\left\{x \in X(\xi) \quad\left(x, \phi_{\xi}(x)\right) \in B\right\}
$$

It is not hard to see that the family $\left\{\pi_{\xi} \xi \in \hat{X}\right\}$ will have to have the following properties for $\hat{\mu}$-a a $\xi$

(60) $\forall$ finite cylinders $E \subset X \times Y$, the mapping $\xi \mapsto \pi_{\xi}(E \cap(X(\xi) \times Y(\xi))$ is $\hat{\mu}$ measurable

(6 1) shift invariance $\pi_{\hat{\sigma}(\xi)}=(\sigma \times \tau) \pi_{\xi}$

(6 2) $\pi_{\xi}$ is a superposition, 1 e a joining of $\mu_{\xi}$ and $\nu_{\xi}$

(6 3) each $\pi_{\xi}$ is forgetful, that is $P^{-(\infty, J) \cap I(\xi)} \perp P^{[j \infty) \cap I(\xi)} Q^{[j, \infty) \cap I(\xi)}\left(\pi_{\xi}\right)$

(64) the marginal $\pi_{\xi}^{*}$ of $\pi_{\xi}$ on $A^{I(\xi)}$ depends only on $\xi[0, \infty)$

(6 3) is a consequence of the fact that $P^{[, \infty) \cap I(\xi)} \supset Q^{[, \infty) \cap I(\xi)}\left(\pi_{\xi}\right)$, which follows from the forgetful nature of $\phi(64)$ is likewise a consequence of the fact that $\phi$ is forgetful However, certainly neither (63) nor (64) implies that $\left\{\pi_{\xi}\right\}$ arises from a mapping consider, for example, $\pi_{\xi}=\mu_{\xi} \times \nu_{\xi}$

The facts that $\phi_{\xi}$ is a forgetful homomorphism and is a e one-to-one are expressed by

$$
Q^{\{0\} \cap l(\xi)} \subset P^{[0 \infty) \cap I(\xi)} \quad\left(\pi_{\xi}\right)
$$

and

$$
P^{(0) \cap J(\xi)} \subset Q^{\prime(\xi)} \quad\left(\pi_{\xi}\right)
$$

(Of course these are vacuous when $0 \notin I(\xi)$ ) Conversely one checks that any family $\left\{\pi_{\xi} \quad \xi \in \hat{X}\right\}$ satısfying (1)-(vi) arıses from a $\phi$ as in Theorem 2 In checkıng that $\phi$ is forgetful (64) is essential if we did not have it we might need the full marker sequence $\xi$ to determıne $\pi_{\xi}^{*}$, which is needed to determıne $\phi(x)(0)$ from $x[0, \infty)$ Accordingly, we turn our attention from mappings to joinings satisfying $(60)$ (66) Let $M_{\xi}$ denote the space of Borel probability measures on $X(\xi) \times Y(\xi)$. We 
denote by $M$ the space of $\hat{\mu}$-equivalence classes of functions $\pi \hat{X} \rightarrow \bigcup\left\{M_{\xi} \quad \xi \in \hat{X}\right\}$, denoted $\xi \mapsto \pi_{\xi}$, such that $\pi_{\xi} \in M_{\xi}$ and (60)-(64) hold for $\hat{\mu}$-a a $\xi$ Note that $M$ is not empty let $\pi_{\xi}=\mu_{\xi} \times \nu_{\xi}$

For $\xi \in \hat{X}$ we define a complete metric $d_{\xi}$ on $M_{\xi}$ which induces the weak-* topology on $M_{\xi}$ as follows Let $\Gamma_{\imath}$ denote the set of all cylinder sets in $X \times Y$ depending only on coordinates in $[-t, 1]$ For $B \subset X \times Y$ let $B_{\xi}=B \cap X(\xi) \times Y(\xi)$ and for $m^{1}, m^{2} \in M_{\xi}$ let

$$
d_{\xi}\left(m^{1}, m^{2}\right)=\sum_{i=0}^{\infty} 2^{-1} \sup _{C \in \Gamma_{t}}\left|m^{1}\left(C_{\xi}\right)-m^{2}\left(C_{\xi}\right)\right|
$$

Now we define a complete metric $d$ on $M$ by

$$
d\left(\pi^{1}, \pi^{2}\right)=\int_{\hat{x}} d_{\xi}\left(\pi_{\xi}^{1}, \pi_{\xi}^{2}\right) d \mu(\xi)
$$

Thus $\pi^{1}$ and $\pi^{2}$ are close if $\pi_{\xi}^{1}$ and $\pi_{\xi}^{2}$ are weak-* close with high $\hat{\mu}$-probability

We introduce approximate versions of $(65)$ and $(66)$ by defining

and

$$
\mathscr{U}_{\varepsilon}=\left\{\pi \in M \quad \hat{\mu}\left\{\xi Q^{\{0\} \cap I(\xi)} \stackrel{\varepsilon}{\subset} P^{[0 \infty) \cap I(\xi)}\left(\pi_{\xi}\right)\right\}>1-\varepsilon\right\}
$$

$$
\mathscr{V}_{\varepsilon}=\left\{\pi \in M \quad \hat{\mu}\left\{\pi P^{\{0\} \cap I(\xi) \varepsilon} Q^{I(\xi)} \quad\left(\pi_{\xi}\right)\right\}>1-\varepsilon\right\}
$$

It is straightforward to check that $\mathscr{U}_{\varepsilon}$ and $\mathscr{V}_{\varepsilon}$ are open subsets of $M$ and that $\bigcap_{n \geq 1}\left(\mathcal{U}_{1 / n} \cap \mathscr{V}_{1 / n}\right)$ consists precisely of those $\pi \in M$ satisfying (6 5) and (6 6) Thus Theorem 2 is a consequence of the following proposition and the Baire category theorem

\section{Proposition 61 The sets $\mathcal{U}_{\varepsilon}$ and $\mathscr{V}_{\varepsilon}$ are dense in $M$}

Proof We deal with $\mathcal{U}_{\varepsilon}$ first What we have to show is that for any $\pi \in M$ we can find $\tilde{\pi} \in \mathscr{U}_{\varepsilon}$ such that $d_{\xi}\left(\pi_{\xi}, \tilde{\pi}_{\xi}\right)$ is small for most $\xi$ We can ensure that $\tilde{\pi} \in \mathscr{U}_{\varepsilon}$ by ensuring that for most (that is, more than $1-\eta$ in $\hat{\mu}$-measure) $\xi$ we have

$$
Q^{(0 \nmid \cap I(\xi)} \stackrel{\varepsilon}{\complement} P^{[0 \infty) \cap I(\xi)} \quad\left(\tilde{\pi}_{\xi}\right)
$$

(The point is that we will in fact be able to make $\eta$ small independent of $\varepsilon$ )

Roughly speakıng we will produce $\tilde{\pi}_{\xi}$ by combinıng the marginals of $\pi_{\xi}$ over large disjoint finite chunks of $\mathbb{Z}$ using the $*$-product, in a way whıch takes advantage of Proposition 47 to ensure that (1) holds for most $\xi$ We use the structure of $\xi$ to determine the chunks, ensuring shift-invariance of $\tilde{\pi}$ If the chunks are sufficiently large then for most $\xi$ a large interval $[-n, n]$ of $\mathbb{Z}$ will be contained in a single chunk so that $\tilde{\pi}_{\xi}$ will have exactly the same marginal (not just close) as $\pi_{\xi}$ over $[-n, n]$, whence $d_{\xi}\left(\pi_{\xi}, \tilde{\pi}_{\xi}\right)$ is small

To this end let $N_{1}<N_{2}, C$ and $m$ be integers to be specified later Suppose $\xi \in \hat{X}$ and $I=[l, J] \subset \mathbb{Z}$ is an interval such that

$$
\xi(l-1)=0 \quad \xi(l)=\xi(l+1)=\quad=\xi(\jmath)=1 \quad \xi(\jmath+1)=0
$$

$I$ will be called a 1 -marker in $\xi$ if $N_{1} \leq \jmath-t+1 \quad I$ will be called a 2 -marker if $N_{2} \leq \jmath-\imath+1$ (so a 2 -marker is also a 1 -marker) Thus a a $\xi \in \hat{X}$ contain infinitely many 1 -markers and infinitely many 2 -markers By an $t$-skeleton $(l=1$ or 2$)$ in $\xi$ 
we mean the set of all indices $t \in \mathbb{Z}$ such that $\xi(t)=0$ which lie between two successive 1 -markers in $\xi$ (Note that this is formally different from the definition of a 1- or 2-skeleton in $\S 3$ ) Thus for a a $\xi \in \hat{X} I(\xi)$ decomposes disjointly into 2-skeleta each of which decomposes into 1-skeleta If $\gamma$ is a 1-skeleton in $\xi, C(\gamma)$ will denote its $C$ rightmost indices and $C(\gamma)=C(\gamma)-\{\max \gamma\}$

Now fix $\xi \in \hat{X}$ and suppose $\mathscr{S}$ is a 2 -skeleton occurring in $\xi$ whose component 1-skeleta are $\gamma_{t}, \gamma_{t-1}, \quad, \gamma_{0}$ listed in order from left to right We set

$$
I(\mathscr{P})=\mathscr{S}
$$

and

$$
J(\mathscr{S})=C\left(\gamma_{t}\right) \cup C\left(\gamma_{t-1}\right) \cup \cup C\left(\gamma_{m}\right)
$$

(Thus $J(\mathscr{S})=\varnothing$ if $t<m$ ) For $t \geq m$ let $\pi_{1}$ denote the marginal of $\pi_{\xi}$ on $A^{\gamma_{1}} \times \mathscr{B}^{C\left(\gamma_{1}\right)}$, for $\imath<m$ let $\pi$, denote $\mu_{\gamma_{1}}$ and let

$$
\begin{array}{rlrl}
\hat{\pi}_{\mathscr{S}} & =\pi_{t} * \pi_{t-1} * & * \pi_{m} * \pi_{m-1} * \quad * \pi_{0} \\
& =\pi_{t} * \pi_{t-1} * & * \pi_{m} * \mu_{\gamma_{m-1} \cup} \cup \gamma_{0}
\end{array}
$$

Next we 'fill in the holes' of $\hat{\pi}_{\mathscr{P}}$ by lettıng $J^{\prime}=\mathscr{S}-J(\mathscr{S})$ and defining

$$
\tilde{\pi}_{\mathscr{S}}=\hat{\pi}_{\mathscr{S}} \times \nu_{J^{\prime}},
$$

a superposition on $A^{\mathscr{S}} \times \mathscr{B}^{\mathscr{S}}$ Finally if

$$
, \mathscr{S}_{-1}, \mathscr{S}_{0}, \mathscr{S}_{1}, \mathscr{S}_{2}
$$

denote the 2-skeleta of $\xi$, listed in order of appearance, we set

$$
\tilde{\pi}_{\xi}=\tilde{\pi}_{\mathscr{S}_{-1}} \times \tilde{\pi}_{\mathscr{S}_{0}} \times \tilde{\pi}_{\mathscr{S}_{1}} \times \tilde{\pi}_{\mathscr{S}_{2}}
$$

(It is not crucial that we take the product joinıng here In fact, roughly speakıng, any 'canonical' joinıng of the $\pi_{\mathscr{S}}^{\prime}$, which is a superposition, for example

$$
\tilde{\pi}_{\mathcal{J}_{-1}} * \tilde{\pi}_{\mathscr{S}_{0}} * \tilde{\pi}_{\mathscr{S}_{1}} \quad \text { would do ) }
$$

It is not difficult to check using Lemmas 42 and 44 that $\tilde{\pi} \in M$ We mention only that (64) is a consequence of (6 3), Lemma 45 and the fact that all our constructions are made workıng from right to left

Moreover, given $\eta>0$ and $k \in \mathbb{Z}^{+}$, If $N_{1}$ is sufficiently large then, with $\hat{\mu}$-probability more than $1-\eta, 0$ is enclosed in a 1-skeleton $\gamma=\gamma(\xi)$ of $\xi$, that is

$$
0 \in[\min \gamma(\xi), \max \gamma(\xi)]
$$

and $\gamma(\xi) \supset[-k, k+1] \cup I(\xi)$ If we next choose $C$ sufficiently large then with high $\hat{\mu}$-probability $C(\gamma(\xi))=\gamma(\xi)$ so

$$
C(\gamma(\xi)) \supset[-k, k] \cap I(\xi)
$$

Now assuming $\gamma(\xi)$ 1s defined let $\mathscr{P}(\xi)$ denote the 2-skeleton in $\xi$ containing $\gamma(\xi)$ and let $\mathscr{S}^{*}(\xi)=\mathscr{F}(\xi) \cap[0, \infty)$ Suppose that the 1 -skeleta of $\mathscr{S}(\xi)$ are $\gamma_{t}, \gamma_{t-1}, \quad, \gamma_{0}$ and $\gamma(\xi)=\gamma_{\bar{t}}$ so

$$
\mathscr{S}^{*}(\xi)=\gamma_{i}^{*} \cup \gamma_{\bar{i}-1} \cup \cdot \cup \gamma_{0}
$$

where $\gamma_{\bar{i}}^{*}=\gamma_{\bar{i}} \cap[0, \infty)$ Let $J^{*}(\mathscr{S}(\xi))=J(\mathscr{S}(\xi)) \cap[0, \infty)$ and let $\tilde{\pi}_{f(\xi)}^{*}$ denote the marginal of $\tilde{\pi}_{\xi}$ on $A^{(\mathscr{f *}(\xi)} \times \mathscr{B}^{J^{*}(\mathscr{P}(\xi))}$, which is the same as the marginal of $\tilde{\pi}_{\mathscr{\varphi}(\xi)}$ on this set, where $\tilde{\pi}_{\mathscr{\varphi}(\xi)}$ is defined by (111) with $\mathscr{S}=\mathscr{S}(\xi)$ Lemmas 44 and 45 together 
with the forgetfulness of $\pi_{\xi}$ imply that

$$
\tilde{\pi}_{\mathscr{S}(\xi)}^{*}=\pi_{\bar{t}}^{*} * \pi_{\bar{i}-1} * \quad * \pi_{m} * \mu_{\gamma_{m-1} \cup} \cup \gamma_{0},
$$

where the $\pi_{i}$ are the measures appearing in (11) and $\pi_{\bar{i}}^{*}$ denotes the marginal of $\pi_{\bar{i}}$ on $\boldsymbol{A}^{\gamma_{i}^{*}} \times \boldsymbol{B}^{C\left(\gamma_{\bar{j}}\right) \cap[0 \infty)}$ Recalling that $\pi_{1}$ is a measure on $\boldsymbol{A}^{\gamma_{1}} \times \mathscr{B}^{C\left(\gamma_{1}\right)}, \# C\left(\gamma_{1}\right) \leq C$ and $\#\left(\gamma_{m-1} \cup \cup \gamma_{0}\right) \geq m$, Proposition 47 implies that if we choose $m$ sufficiently large then for all $\xi$ for which $\gamma(\xi)$ is defined we have

$$
\mu_{\varsigma^{* *}(\xi)}\left(\bigcup\left\{p \in P^{\varphi *(\xi)} p \text { is not split by } \tilde{\pi}_{\varphi(\xi)^{*}}\right\}\right)>1-\varepsilon
$$

so

$$
Q^{J^{*}(\mathscr{S}(\xi))} \stackrel{\varepsilon}{\subset} P^{\mathscr{S}^{*}(\xi)} \quad\left(\tilde{\pi}_{\mathscr{S}(\xi)}^{*}\right)
$$

When the partitions are considered as partitions of $A^{I(\xi)} \times \mathscr{B}^{J(\xi)}$ this implies

$$
Q^{J^{*}(\mathscr{S}(\xi))} \stackrel{\varepsilon}{\subset} P^{l(\xi) \cap[0, \infty)} \quad\left(\tilde{\pi}_{\xi}\right)
$$

Finally, if $N_{2}$ is chosen sufficiently large then with high $\hat{\mu}$-probability $\gamma(\xi)$ is not among the rightmost $m$ 1-skeleta in $\mathscr{S}(\xi)$ so $C(\gamma(\xi)) \subset J(\mathscr{P}(\xi))$ Recalling the definition of $\tilde{\pi}_{\mathscr{S}(\xi)}$ this implies that with high probability $\tilde{\pi}_{\xi}$ and $\pi_{\xi}$ have the same marginal on $A^{\gamma(\xi)} \times \mathscr{B}^{C(\gamma(\xi))}$ In view of (1v) this implies that $d_{\xi}\left(\tilde{\pi}_{\xi}, \pi_{\xi}\right)$ is small Moreover (1v) together with $C(\gamma(\xi)) \subset J(\mathscr{S}(\xi))$ implies that $\{0\} \cap I(\xi) \subset J^{*}((\xi))$ so in view of (v) we obtain that with high $\hat{\mu}$-probability

$$
Q^{\{0\} \cap I(\xi)} \stackrel{\varepsilon}{\subset} P^{I(\xi) \cap[0 \infty)} \quad\left(\tilde{\pi}_{\xi}\right),
$$

as desired This concludes the proof that $\boldsymbol{U}_{\varepsilon}$ is open

To show that $\mathscr{V}_{\varepsilon}$ is open we proceed as follows Fixing $\pi \in M$ we seek $\tilde{\pi} \in \mathscr{V}_{\varepsilon}$ which is close to $\pi$ Let $N_{1}<N_{2}, C, m$ and $M$ be positive integers to be specified later F1X $\xi \in \hat{\boldsymbol{X}}$ and define 1- and 2-skeleta in $\xi$ as before, as well as $\mathscr{S}(\xi)$ and $\gamma(\xi)$ For $\gamma$ a 1-skeleton $C(\gamma)$ and $C(\gamma)$ are also defined as before Suppose $\mathscr{S}$ is a 2-skeleton in $\xi$ with component 1-skeleta $\gamma_{t}, \gamma_{t-1}, \quad, \gamma_{0}$ For $0 \leq t \leq t$ let

$$
\imath=q(M+m)+r(l) \quad 0 \leq r(\imath)<M+m
$$

Call $\gamma_{t}$ principal if $0 \leq r(t)<M$ and auxiliary if $M \leq r(t)<M+m$ Define

and

$$
I(\mathscr{S})=\bigcup\left\{C\left(\gamma_{1}\right) \quad \gamma_{1} \text { principal }\right\}
$$

$$
\pi_{1}=\left\{\begin{array}{l}
\text { marginal of } \pi_{\xi} \text { on } A^{C\left(\gamma_{1}\right)} \times \mathscr{B}^{\gamma_{1}}, \text { if } \gamma_{t} \text { principal } \\
\nu_{\gamma_{1}}, \text { if } \gamma_{1} \text { auxiliary }
\end{array}\right.
$$

Next let

$$
\hat{\pi}_{c \rho}=\pi_{t} * \pi_{t-1} * \quad * \pi_{0},
$$

a superposition on $A^{\left({ }^{(\mathscr{C})}\right.} \times \mathscr{B}^{\mathscr{P}}$, and fill in the holes of $\hat{\pi}_{\mathscr{\varphi}}$ by defining

$$
\tilde{\pi}_{\mathscr{P}}=\hat{\pi}_{\mathscr{S}} \times \mu_{\mathscr{P}-I(\mathscr{f})},
$$

a superposition on $A^{\mathscr{S}} \times \mathscr{B}^{\mathscr{f}}$ Finally if

$$
\mathscr{S}_{-1}, \mathscr{I}_{0}, \mathscr{S}_{1}, \mathscr{S}_{2}
$$

are the component 2-skeleta of $\xi$ set

$$
\tilde{\pi}_{\xi}=\times \tilde{\pi}_{\mathscr{c}_{-1}} \times \tilde{\pi}_{\mathscr{P}_{0}} \times \tilde{\pi}_{\mathscr{f}_{-1}} \times
$$

We can now argue, much as for $\mathscr{U}_{F}$, that $\tilde{\pi} \in \mathscr{V}_{F}$ and is close to $\pi N_{1}, C, m, M$ and $N_{2}$ are chosen in that order The only significantly different feature here is how 
we ensure that for most $\xi$

$$
P^{\{0\} \cap I(\xi)} \stackrel{\varepsilon}{\subset} Q^{I(\xi)} \quad\left(\tilde{\pi}_{\xi}\right)
$$

We begin, as with $U_{\varepsilon}$, by choosing $N_{1}$ so large that for most $\xi \gamma(\xi)$ is defined After $C$ and $m$ are chosen (we will have something to say about them later) if we choose $M$ and then $N_{2}$ sufficiently large then, for most $\xi, \gamma(\xi)$ lies in a string of $M$ consecutive principal 1-skeleta $\gamma_{t+M-1}, \gamma_{t+M-2}, \quad, \gamma_{1}$ of $\mathscr{S}(\xi)$ which is preceded by a strıng of $m$ auxiliary 1-skeleta $\gamma_{1+M+m-1}, \quad, \gamma_{1+M}$ For such a $\xi$ we set

$$
\beta(\xi)=\gamma_{J} \cup \gamma_{\jmath-1} \cup \cup \gamma_{t+M} \cup \gamma_{t+M-1} \cup \cup \gamma_{i},
$$

where $\jmath=\imath+M+m-1$ and

$$
I(\beta(\xi))=I(\mathscr{S}(\xi)) \cap \beta(\xi)=C\left(\gamma_{1+M-1}\right) \cup \quad \cup C\left(\gamma_{1}\right)
$$

Now by associativity of $*$ the marginal of $\tilde{\pi}_{\xi}$ on $A^{I(\beta(\xi))} \times B^{\beta(\xi)}$, which we denote $\tilde{\pi}_{\beta(\xi)}$, has the form

$$
\begin{aligned}
\tilde{\pi}_{\beta(\xi)} & =\left(\pi_{j} * \pi_{j-1} * \quad * \pi_{1+M}\right) * \pi_{1+M-1} * \quad * \pi_{1} \\
& =\nu_{\gamma_{j} \cup} \cup \gamma_{t+M} * \pi_{1+M-1} * \quad * \pi_{1}
\end{aligned}
$$

where the $\pi_{1}$ are the measures appearing in (v1) Thus Lemma 48 guarantees that if we chose $m$ sufficiently large then, regardless of how $M$ and $N_{2}$ were subsequently chosen,

so

$$
P^{I(\beta(\xi))} \stackrel{\varepsilon}{\complement} Q^{\beta(\xi)} \quad\left(\tilde{\pi}_{\beta(\xi)}\right)
$$

$$
P^{I(\beta(\xi))} \stackrel{\varepsilon}{\subset} Q^{I(\xi)} \quad\left(\tilde{\pi}_{\xi}\right)
$$

Now, as in the argument for density of $U_{\varepsilon}$, the choice of $N_{1}$ and $C$ could have been made to ensure that for most $\xi$

$$
C(\gamma(\xi)) \supset[-k, k] \cap I(\xi)
$$

which ensures the closeness of $\pi_{\xi}$ and $\tilde{\pi}_{\xi}$ for most $\xi$ As we already have that $\gamma(\xi)$ is principal for most $\xi$ it also ensures that $\{0\} \cap I(\xi) \subset I(\beta(\xi))$ for most $\xi$ so (v111) implies that (vi1) holds for most $\xi$

\section{REFERENCES}

[B, R] R Burton \& A Rothstein Isomorphism theorem in ergodic theory Preprint

[J] A del Junco Finitary codes between one-sıded Bernoull shifts Ergod Th \& Dynam Sys 1 (1981), 285-301

[K,S] M Keane \& M Smorodinsky Bernoullı shıfts of the same entropy are finitarily isomorphıc Ann Math 109 (1979), 397-406

[M] L Meshalkın A case of isomorphy of Bernoullı schemes Dokl Akad Nauk SSSR 128 (1959), 41-44

[O] D Ornstein Ergodic Theory, Randomness and Dynamical Systems New Haven and London, Yale Unıversity Press (1974)

$[\mathbf{O}, \mathbf{W}]$ D Ornsteın \& B Weıss Unılateral codings of Bernoullı systems Israel J Math 21 (1975), $159-166$

[P] J Propp PhD dissertation, University of Calıfornıa, Berkeley (1987)

[S] Ja G Sinaı A weak isomorphism of transformatıons with an invariant measure Dokl Akad Nauk SSSR 147 (1962), 797-800

[W] P Walters Some results on the classification of non-ınvertible measure-preserving transformations Lecture Notes in Mathematics 318, pp 266-276 Sprınger Verlag, Berin (1973) 